\title{
COMPOSICIÓN ARTEFACTUAL DE LOS CONJUNTOS DE SUPERFICIE DE LA CUENCA DEL LAGO SALITROSO (SANTA CRUZ, ARGENTINA)
}

\author{
GISELA CASSIODORO
}

\begin{abstract}
RESUMEN
La cuenca del lago Salitroso (Santa Cruz) se caracteriza por la existencia de un gran número de sitios a cielo abierto, tanto estructuras de entierros humanos como concentraciones de material arqueológico en superficie. En esta oportunidad se presenta la información concerniente a la composición artefactual de estas concentraciones y los espacios entre ellas. Los conjuntos se localizan principalmente en sectores de médanos y las cronologías disponibles los ubican en momentos posteriores a ca. 1.600 años AP. Así, se reseñan las características de la diversidad artefactual, las clases artefactuales representadas y las materias primas utilizadas. A partir de un análisis tecno-morfológico y cuantitativo se busca dar cuenta de la variabilidad tecnológica, la cual es explicada en el marco del proceso de descenso de la humedad regional del Holoceno tardío. Durante el mismo se ha propuesto que la cuenca lacustre baja, en estudio, habría sido utilizada de manera permanente y semi-permanente de forma residencial por parte de los grupos cazadores- recolectores (Goñi 2000).
\end{abstract}

PALABRAS CLAVES: cazadores- recolectores, cuenca lacustre, tecnología, movilidad, holoceno tardío.

\section{ARTEFACTUAL COMPOSITION OF SURFACE ASSEMBLAGES IN THE SALITROSO LAKE BASIN (SANTA CRUZ, ARGENTINA)}

\begin{abstract}
The Salitroso lake basin (Santa Cruz) is characterized by the existence of a great number of open air sites, both funerary structures and surface concentrations of archaeological materials. In this opportunity the information regarding the artefactual composition of these concentrations and the spaces between them is presented. The assemblages are located in dunes and the available chronologies date them after 1.600 years BP. The characteristics of artefactual diversity, types and raw materials are presented. Technological
\end{abstract}


variability is discussed by means of a techno-morphological and quantitative analysis. This variability is explained within the framework of the late Holocene regional humidity drop. It is proposed that during this process the lake basin under study would have been used permanently and semi-permanently in a residential manner by hunter-gatherer groups (Goñi 2000).

KEYWORDS: hunter- gatherers, lake basin, technology, mobility, late Holocene.

\section{INTRODUCCIÓN}

La cuenca de los lagos Salitroso-PosadasPueyrredón, localizada al noroeste de la provincia de Santa Cruz, se caracteriza por ser un gran bajo con alturas que van de los 100 a los 300 m.s.n.m. Es un ambiente de estepa arbustiva (Cabrera y Willink 1980) que ofrece buenas pasturas, leña, agua disponible, baja carga de nieve invernal, posibilidades de reparo (tanto en formaciones rocosas como en médanos) y recursos animales diversos. En particular, la cuenca endorreica del lago Salitroso abarca aproximadamente unos $280 \mathrm{~km}^{2}$ que van desde la margen oriental del lago hasta la Estancia Río Blanco.

El modelo de poblamiento propuesto para la cuenca se apoya en la existencia de cambios climático y ecológicos regionales durante el Holoceno tardío (Goñi 2000; Goñi et al. 2000-2002; Goñi y Barrientos 2004). Los mismos consistirían en un proceso de progresiva, aunque fluctuante, desecación ambiental que habría comenzado hacia los 2.500/2.000 años AP (Stine y Stine 1990; González 1992) e intensificado hacia los 900 años AP con el desarrollo de la denominada Anomalía Climática Medieval (Stine 1994, Stine 2000). Estas condiciones habrían provocado un cambio en la estructura de recursos. Principalmente, se vería afectada la existencia y el tamaño de los cuerpos de agua. Por otro lado, dado el carácter marcadamente estacional de Patagonia, la disponibilidad de leña y de condiciones ambientales menos hostiles durante los meses de mayor frío se tornan fundamentales. En consecuencia, la localización de más de un recurso crítico en un área aumentaría la probabilidad de su uso y reuso (Ebert y Kohler 1988). De esta manera, las cuencas lacustres con disponibilidad de este tipo de recursos y condiciones se habrían convertido en espacios privilegiados para la ocupación humana. Así, los sectores con estas características, como es el caso de la cuenca en estudio, serían potencialmente utilizables durante todo el año, llevando a la reducción de la movilidad residencial de los grupos cazadores- recolectores (Goñi 2000). Este proceso de reducción de la movilidad residencial, ausente en momentos previos, habría llevado al nucleamiento de poblaciones en determinados sectores ambientales, a partir de los cuales se desarrollarían movimientos logísticos y estacionales hacia otros sectores para la obtención de recursos específicos, como cuencas lacustres altas o las mesetas (Goñi 2000; Goñi et al. 2000-2002; Goñi y Barrientos 2004).

Considerando que la tecnología debería reflejar los cambios mencionados, las investigaciones tecnológicas del área buscan explicar cuáles serían las características de la utilización de distintos ambientes bajo condiciones de mayor sequedad regional y cuáles serían sus implicancias tecnológicas. Es decir, cuáles serían los aspectos tecnológicos desarrollados en momentos donde, por distintos factores, la movilidad residencial de los grupos se vería reducida (Cassiodoro 2008).

En la cuenca del lago Salitroso se ha determinado la existencia de estructuras de acumulación de piedras de forma oval o circular con entierros humanos (chenques n: 34), estructuras con características similares pero sin entierros (n: 9) y estructuras con entierros que combinan la utilización de oquedades naturales con la acumulación de rocas (entierros bajo bloque n: 4). A su vez, existen inhumaciones que no hacen uso de estructuras de piedra sino de formaciones naturales (nichos n: 6). Al mismo tiempo, existen conjuntos artefactuales registrados tanto en concentraciones como de manera aislada. Así, se han relevado 19 concentraciones de material lítico, cerámico y óseo. Los fechados radiocarbónicos obtenidos, tanto para las concentraciones

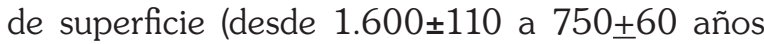
AP) como para los enterratorios (desde $2.607 \pm 41$ a $352 \pm 40$ años AP), sitúan a las ocupaciones humanas en un período climático seco del Holoceno tardío (Goñi 2000-2002; Goñi et al. 2000-2002; Goñi y Barrientos 2004; Cassiodoro et al. 2004; 
García Guráieb et al. 2007a y b; Cassiodoro y García Guráieb 2009).

Por su parte, en la cuenca del lago Posadas, el alero Cerro de los Indios 1 cuenta con cronolo-

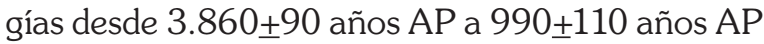
agrupadas en dos bloques temporales (Aschero et al. 1999; De Nigris et al. 2004) y las concentraciones de material arqueológico en superficie (Médanos del Lago Posadas y Lago Posadas Entrada Hostería) tienen cronologías entre los 560 \pm 90 años AP y los 770 \pm 30 años AP (Goñi 2000-2002).

\section{OBJETIVOS}

El objetivo principal de las investigaciones tecnológicas en el área es evaluar la movilidad de los grupos cazadores-recolectores del noroeste de la provincia de Santa Cruz en el marco de las condiciones climático/ ambientales del Holoceno tardío (Cassiodoro 2008). En particular, el objetivo general del trabajo es establecer las características de los aspectos tecnológicos de la cuenca del lago Salitroso, abordando el estudio de las materias primas utilizadas y la estructura artefactual de los conjuntos. Se maneja como hipótesis que las características de los conjuntos tecnológicos estarán condicionadas por el carácter residencial y la mayor duración de las ocupaciones en las cuencas lacustres bajas, en un contexto de reducción de la movilidad durante el Holoceno tardío. Por lo tanto, se espera que en la cuenca del lago Salitroso:

- Todos los sectores hayan sido frecuentemente utilizados, por lo cual el registro tecnológico se distribuirá de manera continua y densa en el espacio.

- La diversidad artefactual de los conjuntos sea alta en función del desarrollo de un amplio espectro de actividades.

- Existan estrategias de equipamiento de espacios con una alta frecuencia de elementos tecnológicos con diseños poco transportables o no transportables.

- Se registre una alta diversidad de materias primas utilizadas, considerando la disponibilidad de recursos minerales en el área, la mayor permanencia en la ocupación, la existencia de movimientos logísticos y el tipo de tareas efectuadas.

Para evaluar estas expectativas, en esta oportunidad se presenta la información concerniente a la composición artefactual de las concentraciones de material arqueológico y las características del material que se encuentra de forma aislada. Así, se reseñan las clases artefactuales representadas, las características de la diversidad artefactual (riqueza y homogeneidad) de los conjuntos y las materias primas utilizadas. A partir de un análisis tecno-morfológico y cuantitativo se busca dar cuenta de la variabilidad tecnológica de las concentraciones de material arqueológico para discutir el carácter de las actividades desarrolladas en las mismas. Dicha variabilidad es explicada en el marco del proceso de descenso de la humedad regional del Holoceno tardío.

El acercamiento a la variabilidad de la tecnología se realiza guiado por lineamientos teóricos generales que se vinculan, principalmente, con una perspectiva ecológico- evolutiva que plantea que la interacción con el medioambiente físico y social es un hecho fundamental de la vida humana (Winterhalder y Smith 1992). En particular, la tecnología ha sido abordada desde un acercamiento organizativo, buscando relacionar los artefactos con aspectos económicos y sociales de los grupos (Binford 1979; Carr 1994).

\section{DISTRIBUCIÓN, FRECUENCIA Y DENSIDAD}

Dado el carácter regional de las investigaciones se han realizado diferentes tipos de relevamientos para dar cuenta de las propiedades del registro arqueológico de la cuenca del lago Salitroso. La estrategia de muestreo buscó relevar todos los sectores de la misma. A partir de estos trabajos fueron consignados una serie de puntos en el espacio que presentaban estructuras de entierro o correspondían a concentraciones de material arqueológico en superficie (figura 1).

En la siguiente tabla se sintetizan los aspectos referidos a la distribución, frecuencia y densidad del registro de las concentraciones artefactuales mencionadas (tabla 1). Se presenta la nomenclatura de estos sectores, la cota y la geoforma en la que se encuentran. La tabla también resume la información sobre la frecuencia de materiales tecnológicos y la densidad de artefactos líticos en cada uno de los relevamientos. Así, se especifica la superficie muestreada en cada uno de las concentraciones y la cantidad de artefactos líticos recuperados en la misma. Se debe aclarar que en la superficie muestreada no se incluyen los sondeos y excavaciones, 


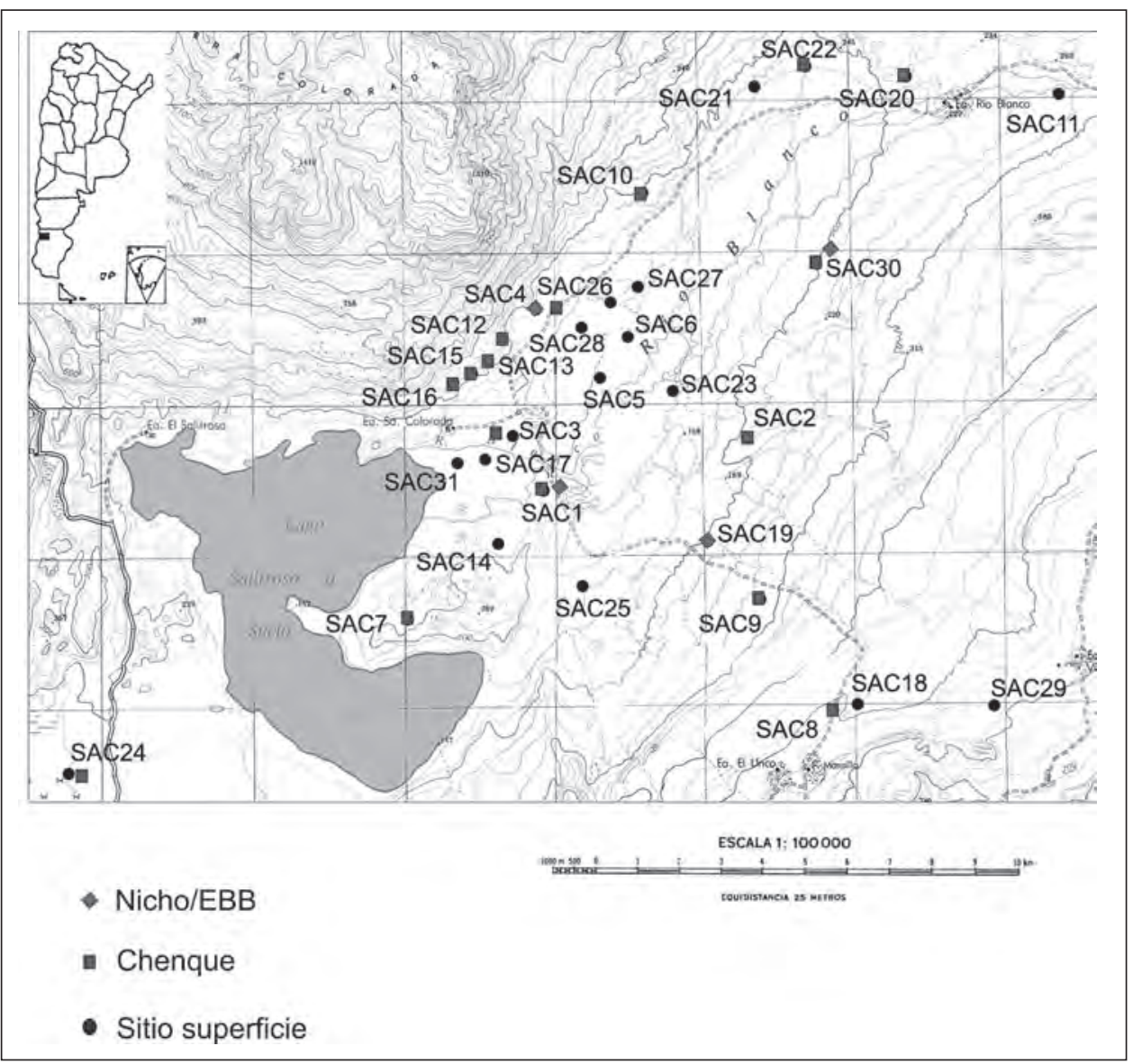

Fig. 1. Area de estudio.

es por ello que tampoco se incluye los materiales recuperados en ellos. Los sondeos que arrojaron material lítico fueron los realizados en SAC 3, SAC 11 y SAC 25. En la tabla además se especifican las frecuencias de otras tecnologías registradas (cerámica $y$ artefactos en hueso).

Se observa que la mayoría de las concentraciones se encuentran localizadas en el fondo de cuenca, en médanos, con densidades artefactuales variables. A su vez, se destaca la presencia de cerámica en varias de las mismas. El fechado disponible para SAC 3 es de $960 \pm 125$ años AP (Goñi 2000-2002). En las inmediaciones de la concentración de SAC 4 se localiza un chenque con un fechado de 728 \pm 39 años AP, un entierro bajo bloque con un fechado de 2.274 \pm 41 años AP y seis enterratorios en nichos con cronologías de $2.607 \pm 41$ y $2.520 \pm 40$ años AP (Goñi et al. 2000-2002; Bernal et al. 2004; García Guráieb et al. 2007a). En la concentración de SAC
11 se obtuvo un fechado radiocarbónico de 750+60 años AP (Goñi 2000-2002) y en la de SAC 25 uno de $1.600 \pm 100$ años AP (Cassiodoro 2008).

Teniendo en cuenta los sectores con chenques y concentraciones de material arqueológico en superficie se establecieron prospecciones y transectas para evaluar la distribución del registro arqueológico entre los mismos y evitar sesgos de muestreo tendientes a focalizarse en la localización de las concentraciones artefactuales o "sitios". A su vez, se buscó establecer la continuidad o no del registro arqueológico en el espacio. En la siguiente tabla se presenta la nomenclatura de las prospecciones y transectas realizadas, la cota, las superficies muestreadas y las características del registro tecnológico (tabla 2).

Puede observarse la baja densidad artefactual registrada en las prospecciones y transectas realizadas, siendo las que se encuentran próximas a concentraciones las que presentan valores superiores. En 
Tabla 1. Distribución, frecuencia y densidad registro de superficie de concentraciones artefactuales.

\begin{tabular}{|c|c|c|c|c|c|c|c|}
\hline Nombre & Cota & Geoforma & $\begin{array}{l}\text { Superficie } \\
\text { mues. }\left(\mathrm{m}^{2}\right)\end{array}$ & Frec. art.lit. & $\begin{array}{c}\text { Frec. } \\
\text { Cerámica }\end{array}$ & $\begin{array}{l}\text { Frec. } \\
\text { art. hue. }\end{array}$ & $\begin{array}{c}\text { Densidad } \\
\text { art. lit. }\end{array}$ \\
\hline SAC3 & $150-200$ & médano & 198,5 & 609 & 4 & - & 3,06 \\
\hline SAC4 & $150-200$ & cerro & 36000 & 363 & - & - & 0,01 \\
\hline SAC5 & $150-200$ & médano & 2700 & 501 & 35 & - & 0,185 \\
\hline SAC6 & $150-200$ & médano & 50 & 191 & 51 & 1 & 3,82 \\
\hline SAC7 & $200-300$ & terraza & 3500 & 46 & - & - & 0,013 \\
\hline SAC10 & $150-200$ & abanico & 8400 & 232 & - & - & 0,027 \\
\hline SAC11 & $200-300$ & médano & 450 & 1023 & 173 & - & 2,273 \\
\hline SAC14 & $150-200$ & médano & 20 & 65 & - & - & 3,25 \\
\hline SAC18 & 500 & médano & 158 & 245 & - & - & 1,55 \\
\hline SAC20 & $200-300$ & médano & 10750 & 180 & - & - & 0,016 \\
\hline SAC21 & $150-200$ & abanico & 5000 & 74 & - & - & 0,014 \\
\hline SAC23 & $150-200$ & médano & 2500 & 95 & 4 & - & 0,038 \\
\hline SAC24 & $150-200$ & médano & 150 & 2067 & 10 & - & 13,78 \\
\hline SAC25 & $150-200$ & médano & 159 & 110 & - & - & 0,691 \\
\hline SAC26 & $150-200$ & médano & 6825 & 270 & - & - & 0,039 \\
\hline SAC27 & $150-200$ & médano & 6370 & 169 & - & - & 0,026 \\
\hline SAC28 & $150-200$ & médano & 9604 & 82 & - & - & 0,008 \\
\hline SAC29 & 500 & médano & 208 & 203 & 14 & - & 0,975 \\
\hline SAC31 & $150-200$ & médano & 504 & 53 & - & - & 0.1 \\
\hline
\end{tabular}

Referencias: mues.: muestreada, frec.: frecuencia, art. lit.: artefactos líticos, hue.: hueso.

estos relevamientos sólo se observó material lítico y se evidencia la continuidad espacial del registro arqueológico.

Como ha sido mencionado en otras oportunidades (Goñi y Barrientos 2000; Goñi et al. 2000-2002; Cassiodoro et al. 2004), el registro arqueológico de la cuenca del lago Salitroso se distribuye tanto en sectores bajos o fondo de cuenca vinculados con médanos y abanicos aluviales como en sectores más altos relacionados con bardas, cerro y terrazas glacilacustres, implicando una alta densidad de artefactos y estructuras de piedra en un espacio que abarca unos $280 \mathrm{~km}^{2}$. A su vez, el registro se distribuye de forma diferencial en los distintos sectores geomorfológicos especificados. En los sectores bajos de la cuenca se presenta un mayor número de concentraciones artefactuales o puntos del espacio con mayor densidad artefactual. Estos puntos tienden a estar separados entre sí por espacios con una baja densidad artefactual. En contraposición, en los sectores con cotas altitudinales mayores tienden a registrarse las estructuras de piedra (chenques). Al mismo tiempo, existe una asociación entre diferentes aspectos tecnológicos. Así, los tiestos cerámicos están vinculados con los puntos con mayor densidad artefactual y tecnologías en metal, cuentas de vidrio y valvas se relacionan con las estructuras de piedra (Cassiodoro y García Guraieb 2009).

Estos aspectos estarían evidenciando una distribución heterogénea del registro arqueológico en el espacio. Por un lado, la misma se relaciona con las características geomorfológicas del área y, por otro, con los aspectos composicionales del registro. En el siguiente punto se detalla la información referida a la composición del registro arqueológico, principalmente a la tecnología lítica por ser ésta la más representada en la cuenca.

\section{COMPOSICIÓN ARTEFACTUAL}

\section{Estructura artefactual}

En primer lugar, se presenta la totalidad de tipos de artefactos líticos junto con la frecuencia de tiestos cerámicos que han sido registrados en las concentraciones artefactuales. Así, en la tabla 3 se presenta la estructura artefactual de las mismas, incluyendo el total de artefactos recuperados en superficie y estratigrafía. Para los criterios clasificatorios de los artefactos se siguió la metodología propuesta por Aschero (1975 rev. 1983). 
Tabla 2. Distribución, frecuencia y densidad del registro en prospecciones y transectas.

\begin{tabular}{|c|c|c|c|c|}
\hline Nombre & Cota & Superficie muestreada $\left(\mathrm{m}^{2}\right)$ & Frec. art.lit. & $\begin{array}{c}\text { Densidad } \\
\text { art. lit. }\end{array}$ \\
\hline SAC1 BARDA ESTE & $150-200$ & 20370 & 11 & 0,00054 \\
\hline SAC1 BARDA OSTE & $150-200$ & 2025 & 149 & 0,0738 \\
\hline $\mathrm{SAC} 2 \mathrm{CH} 8$ & $200-300$ & 1000 & 49 & 0,049 \\
\hline SAC2/5-SAC30 A & $200-300$ & 15000 & 21 & 0,0014 \\
\hline SAC2/5-SAC30 B & $200-300$ & 40000 & 7 & 0,00017 \\
\hline SAC3 TR 250은 & $150-200$ & 4575 & 15 & 0,0032 \\
\hline SAC3 TR 50은 & $150-200$ & 12000 & 11 & 0,0009 \\
\hline SAC3 TR $130^{\circ}$ & $150-200$ & 7000 & 222 & 0,032 \\
\hline SAC3 TR $310^{\circ}$ & $150-200$ & 7550 & 10 & 0,001 \\
\hline SAC3 TR 350응 & $150-200$ & 1050 & 6 & 0,0057 \\
\hline SAC5 TR 1 & $150-200$ & 4500 & 1 & 0,0002 \\
\hline SAC5 TR 2 & $150-200$ & 4500 & 6 & 0,001 \\
\hline SAC5 TR 3 & $150-200$ & 4500 & 11 & 0,002 \\
\hline SAC5 TR 4 & $150-200$ & 4500 & 42 & 0,009 \\
\hline SAC5 TR 5 & $150-200$ & 4500 & 19 & 0,004 \\
\hline SAC5 TR 6 & $150-200$ & 4500 & - & - \\
\hline SAC25 C2 TR 1 & $150-200$ & 500 & 17 & 0,034 \\
\hline SAC25 C2 TR 2 & $150-200$ & 500 & 4 & 0,008 \\
\hline SAC8/3-SAC29 & 500 & 2940 & - & - \\
\hline SAC29-COTA600 & 500 & 3600 & 7 & 0,0019 \\
\hline SAC1-SAC14 & $150-200$ & 22500 & 27 & 0,0012 \\
\hline LAGO-CHACRA & $150-200$ & 30000 & - & - \\
\hline CHACRA-SAC31 & $150-200$ & 140000 & 15 & 0,0001 \\
\hline CASCO-SAC5 & $150-200$ & 3000 & - & - \\
\hline CASCO-SAC4 & $150-200$ & 3000 & 4 & 0,00013 \\
\hline CASCO-SAC3 & $150-200$ & 1000 & - & - \\
\hline CASCO-SAC2/5 & $150-200$ & 105000 & - & - \\
\hline SAC10-RIO & $150-200$ & 250000 & 6 & 0,000024 \\
\hline RIO-CAMINO & $150-200$ & 58000 & 4 & 0,000068 \\
\hline
\end{tabular}

Referencias: frec.: frecuencia, tr.: transecta, art. lit.: artefactos líticos.

Se observa que la clase artefactual presente en mayor frecuencia corresponde a los desechos de talla. Los artefactos formatizados más representados son los raspadores, principalmente en las concentraciones de SAC 5, SAC 6, SAC 10, SAC 18, SAC 20, SAC 24, SAC 26, SAC 27, SAC 28 y SAC 29. Otros artefactos formatizados presentes en mayores proporciones son los fragmentos de artefactos formatizados, los artefactos de formatización sumaria, las puntas de proyectil y las raederas. También es de destacar la alta frecuencia de núcleos, en especial en las concentraciones SAC 4, SAC 14 y SAC 23.

Cabe destacar diferencias entre los conjuntos principalmente vinculadas con la representación diferencial de algunas clases artefactuales, como la presencia de artefactos de molienda y cerámica en SAC 3, SAC 5, SAC 6, SAC 11, SAC 23, SAC 24, SAC 29.

Aunque no constituyen artefactos líticos, se debe mencionar la presencia de rocas sin formatización, de dimensiones similares a los artefactos de molienda. Las mismas han sido observadas en las concentraciones ubicadas en médanos de SAC 3, SAC 5, SAC 6, SAC 11, SAC 24, SAC 28, SAC 29 y SAC 31. Dado que estas rocas no forman parte del sustrato natural de los médanos, se plantea la posibilidad que hayan sido transportadas intencionalmente a los mismos.

A continuación, se presenta en la tabla 4 la estructura artefactual correspondiente a las prospecciones y transectas. 


\begin{tabular}{|c|c|c|c|c|c|c|c|c|c|c|c|c|c|c|c|c|c|c|c|c|c|c|c|}
\hline $\begin{array}{l}\overline{7} \\
0\end{array}$ & ले & $\vec{\infty}$ & $\stackrel{N}{\sim}$ & \begin{tabular}{|l|}
10 \\
$\stackrel{2}{\circ}$ \\
$\sim$
\end{tabular} & $\tilde{\sigma}$ & $r$ & $\stackrel{\mathfrak{I}}{\mathcal{I}}$ & Fั & 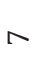 & $\infty$ & ले & $\stackrel{\circ}{\sim}$ & $\stackrel{\text { 足 }}{\stackrel{2}{-}}$ & $\infty$ & $\infty$ & $\underset{\infty}{\infty}$ & $\underset{\sim}{\stackrel{2}{\sim}}$ & $n$ & 음 & $\begin{array}{l}\text { ลे } \\
\text { مิ }\end{array}$ & ๙े & $\begin{array}{l}\infty \\
\infty \\
\stackrel{\infty}{N}\end{array}$ & \\
\hline$\underset{c}{\mathbb{2}}$ & -1 & -1 & ' & -1 & -1 & ' & -1 & -1 & ' & $m$ & $\sim$ & 1 & -1 & ' & -1 & ' & -1 & 1 & ' & 아 & ' & 它 & 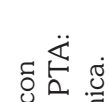 \\
\hline స్ & 6 & $\sigma$ & 1 & $N$ & 10 & ' & + & ' & ' & \llcorner & $N$ & 1 & $a$ & -1 & ' & $\stackrel{\sim}{\sim}$ & $n$ & 1 & $\infty$ & 绐 & $\Xi$ & ָิ & 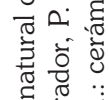 \\
\hline 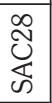 & $m$ & ' & $\sim$ & $m$ & -1 & ' & 1 & ' & -1 & $N$ & ' & 1 & 6 & -1 & ' & $\infty$ & I & -1 & ' & में & ' & $\infty$ & 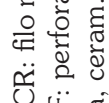 \\
\hline 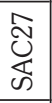 & $\infty$ & + & 1 & $n$ & 10 & ' & $m$ & $m$ & ' & $N$ & ' & 1 & 0 & ' & ' & $a$ & $\stackrel{N}{\sim}$ & 1 & ' & 으 & ' & $\stackrel{\circ}{6}$ & 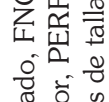 \\
\hline 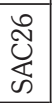 & $\infty$ & $\infty$ & 1 & $m$ & $\infty$ & ' & $\stackrel{m}{\rightarrow}$ & $m$ & - & $N$ & ' & 1 & $\stackrel{\infty}{\sim}$ & -1 & -1 & F & $\stackrel{N}{7}$ & -1 & ' & 守 & -1 & $\stackrel{\vec{N}}{\mathrm{~N}}$ & 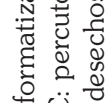 \\
\hline 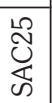 & -1 & -1 & 1 & $\sim$ & -1 & ' & 二 & $N$ & - & 6 & + & 1 & -1 & -1 & 1 & $m$ & $a$ & 1 & -1 & $\underset{\sim}{\sim}$ & ' & $\stackrel{\infty}{\infty}$ & 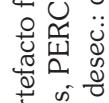 \\
\hline 志 & 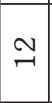 & 0 & $\sim$ & $\exists$ & 0 & ' & $a$ & $N$ & ' & $m$ & ' & 1 & $\approx$ & ' & $N$ & 등 & + & 1 & ' & $\begin{array}{l}0 \\
\infty \\
\curvearrowright \\
\ddots\end{array}$ & 음 & 令 & $\begin{array}{l}\sigma \\
0 \\
0 \\
0 \\
0\end{array}$ \\
\hline 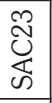 & $\sigma$ & $\sim$ & 1 & -1 & -1 & ' & $N$ & ' & ' & ' & ' & 1 & 10 & -1 & ' & $\sigma$ & $n$ & 1 & -1 & ㅇ & $\sigma$ & $\stackrel{\infty}{\sigma}$ & 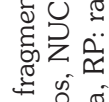 \\
\hline \begin{tabular}{l}
$\overrightarrow{\tilde{N}}$ \\
\multirow{\leftarrow}{*}{}
\end{tabular} & ' & ' & 1 & & ' & & 0 & & 1 & ' & & 1 & $\infty$ & 1 & ' & 0 & $N$ & 1 & & $\stackrel{\infty}{\infty}$ & I & 吾 & I. \\
\hline 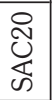 & $\nabla$ & $m$ & 1 & $\sim$ & ' & ' & 1 & $N$ & ' & ' & & 1 & 0 & 1 & ' & $\infty$ & $N$ & 1 & & 总 & ' & 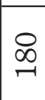 & $\begin{array}{l}\overline{\bar{Z}} \\
\bar{U} \\
\bar{U}\end{array}$ \\
\hline 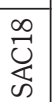 & 0 & + & -1 & $m$ & 1 & ' & $\sigma$ & $N$ & ' & ' & & 1 & 10 & 1 & 1 & 亗 & 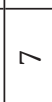 & 1 & & $\overrightarrow{\mathrm{N}}$ & ' & త్ర & స̃ \\
\hline $\begin{array}{l}\overrightarrow{\vec{U}} \\
\stackrel{\mathbb{4}}{\infty}\end{array}$ & $m$ & $N$ & 1 & ' & -1 & ' & 1 & -1 & -1 & -1 & & 1 & 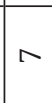 & ' & 1 & $\sim$ & $N$ & 1 & -1 & 字 & ' & 6 & 岗 \\
\hline 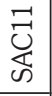 & शे & 0 & $\sim$ & $\stackrel{\sim}{\sim}$ & 岕 & ' & $\stackrel{m}{\sim}$ & 으 & -1 & $\exists$ & -1 & 1 & ๙ & 1 & 1 & $\stackrel{2}{\sim}$ & $\stackrel{m}{-1}$ & 1 & & $\begin{array}{l}\text { §ి } \\
\infty\end{array}$ & $\stackrel{2}{\approx}$ & 号 & हี \\
\hline 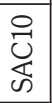 & + & $m$ & . & $\stackrel{m}{\rightarrow}$ & $\sigma$ & ' & $\Lambda$ & $N$ & ' & 6 & - & 1 & $\infty$ & $m$ & 1 & 등 & $m$ & -1 & ' & 임 & ' & స్ & 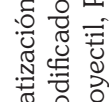 \\
\hline 仓ิ & 1 & $m$ & 1 & ' & ' & -1 & $\sigma$ & 1 & ' & , & 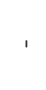 & 1 & & 1 & 1 & 0 & 0 & 1 & 1 & $\stackrel{\sim}{\sim}$ & , & 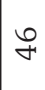 & 竞 \\
\hline 悹 & $\exists$ & $\underline{-1}$ & -1 & $\stackrel{\infty}{+}$ & $a$ & -1 & & $\exists$ & $N$ & ఠ) & $\stackrel{\infty}{\sim}$ & 1 & 2े & ' & $m$ & 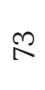 & $\stackrel{\infty}{\sim}$ & 1 & $N$ & के & $\vec{n}$ & 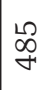 & ?. \\
\hline 岕 & 0 & $\sigma$ & ' & $a$ & $\sigma$ & . & 岕 & -1 & ' & 0 & -1 & 1 & $\underset{\sim}{\sim}$ & ' & 1 & 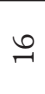 & م & 1 & 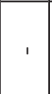 & 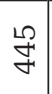 & $\stackrel{\mathscr{m}}{\mathrm{m}}$ & 足 & $\ddot{\ddot{n}}$ \\
\hline 导 & $\exists$ & $\Lambda$ & $N$ & 10 & -1 & ' & $N$ & , & , & ' & ' & $\stackrel{\llcorner}{\sim}$ & ก & 1 & ' & $\stackrel{m}{7}$ & 10 & 1 & -1 & $\overrightarrow{\mathrm{N}}$ & , & $\begin{array}{l}n \\
0 \\
0\end{array}$ & 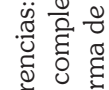 \\
\hline 灾 & $\underline{-1}$ & $\Lambda$ & $N$ & N & $N$ & 10 & 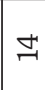 & $N$ & ' & $N$ & & 1 & a & 1 & -1 & $r$ & 10 & 1 & & $\stackrel{20}{\sim}$ & $\sigma$ & $\begin{array}{l}m \\
\infty\end{array}$ & $\approx$ \\
\hline & $\frac{n}{\pi}$ & 节 & $\overrightarrow{\mathrm{z}}$ & 亲 & ق : & है & שֶ, & Ј & $\begin{array}{l}0 \\
\underline{0} \\
\underline{\underline{x}}\end{array}$ & $\begin{array}{l}\text { : } \\
\text { है } \\
\text { है }\end{array}$ & ह & $\begin{array}{l}\dot{0} \\
0 \\
0\end{array}$ & है & ֻू & 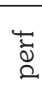 & $\cong$ & D & \begin{tabular}{|l|}
$\frac{\pi}{0}$ \\
8 \\
$\dot{2}$ \\
\end{tabular} & $\frac{\pi}{8}$ & $\begin{array}{l}\dot{0} \\
\mathbb{0} \\
\mathbb{8} \\
0\end{array}$ & 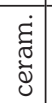 & 胥 & \\
\hline
\end{tabular}




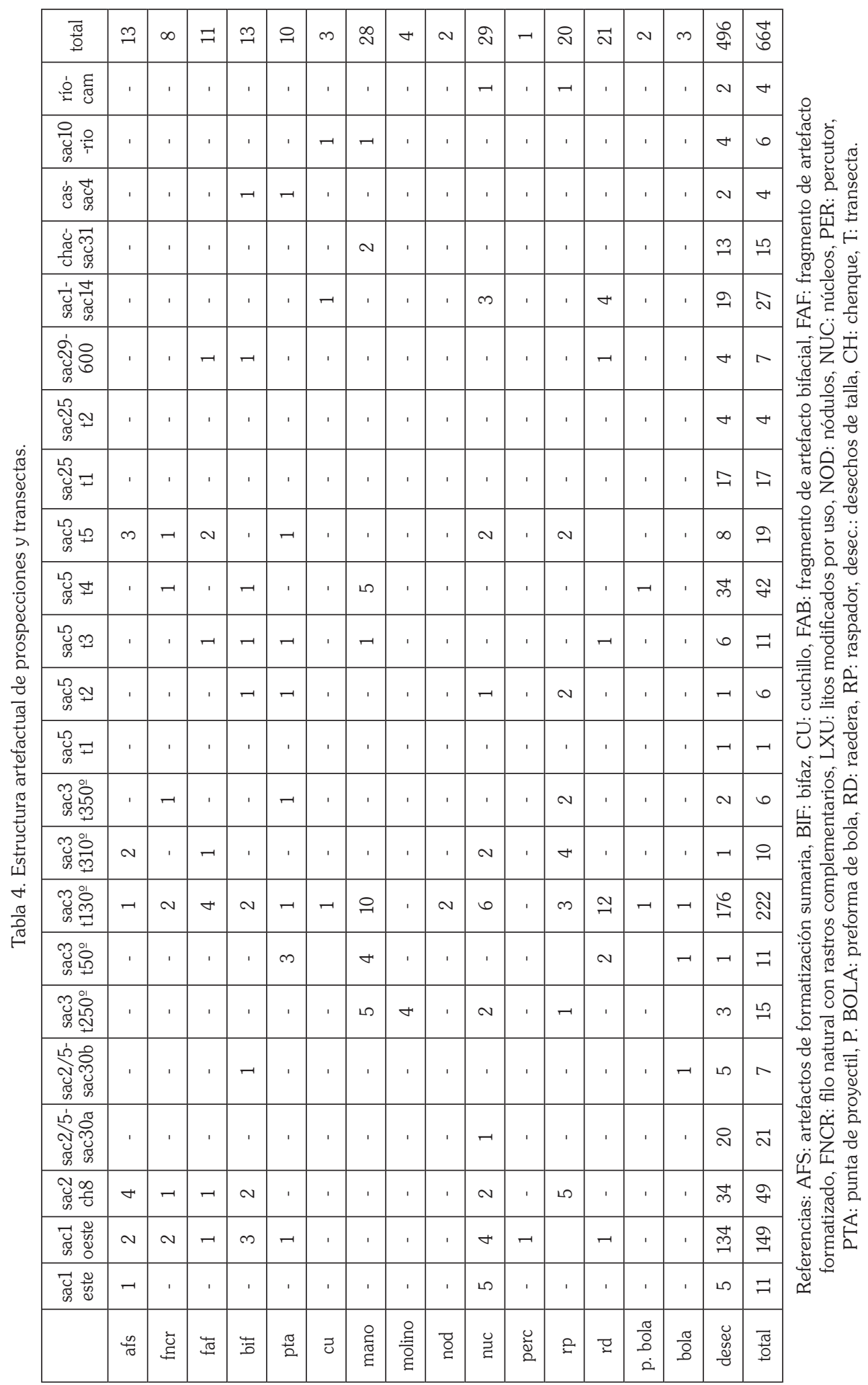


La estructura artefactual relevada en prospecciones y transectas también tiene a los desechos de talla como la clase artefactual más representada. Sin embargo, en este caso los núcleos, raederas y manos se encuentran en mayor frecuencia que los raspadores. Otra diferencia con las concentraciones artefactuales es la ausencia de cerámica y la menor cantidad de clases artefactuales representadas.

Diversidad artefactual

Como parte de la caracterización de la composición de los conjuntos tecnológicos se puede incluir la diversidad de los mismos. La diversidad incluye tres elementos: riqueza, homogeneidad y heterogeneidad (Pielou 1977). Se define a la riqueza artefactual como la cantidad de clases de artefactos representadas en el conjunto y la homogeneidad busca evaluar si todas las clases son igualmente abundantes (Shott 1989; Lanata 1996). Por su parte, la heterogeneidad mide la relación entre el número de clases y la abundancia relativa de cada una de ellas, se ha planteado que el uso de este índice en arqueología no es aconsejable (Lanata 1996). Para medir la riqueza se ha utilizado la fórmula de Shannon y Weaver (1949) y para la homogeneidad la derivada de Zar (1974) y Pielou (1977). En ambos casos no han sido incluidos los desechos de talla y los tiestos cerámicos considerando que ambos podrían estar sobredimensionando una clase. El índice $\mathrm{H}$ de la riqueza establece que cuando todos los artefactos de un conjunto pertenecen a una misma categoría, el valor que asume es 0 . Por su parte, los valores máximos son una función del número de categorías. Por otro lado, el índice de homogeneidad $\mathrm{J}$ toma el valor máximo de 1 cuando todos los elementos se encuentran repartidos equitativamente en las categorías artefactuales (Guráieb 1999). Como mencionan otros autores estos índices son afectados por los criterios clasificatorios aplicados a las muestras (Shott 1989; Lanata 1996; Guráieb 1999; Álvarez 2004). La información de la diversidad artefactual de las concentraciones se presenta en la siguiente tabla (tabla 5).

En líneas generales existe una alta riqueza artefactual en las concentraciones de superficie del lago Salitroso, con valores de $\mathrm{H}$ superiores a 0,85 , siendo los conjuntos de SAC 29, SAC 31, SAC 6 y SAC 11 los más ricos. No obstante, también existen
Tabla 5: riqueza y homogeneidad artefactual concentraciones artefactuales.

\begin{tabular}{|c|c|c|c|c|}
\hline & $\mathbf{n}$ & $\mathbf{k}$ & $\mathbf{H}$ & $\mathbf{J}$ \\
\hline SAC3 & 94 & 13 & 0.966 & 0.867 \\
\hline SAC4 & 92 & 11 & 0.865 & 0.831 \\
\hline SAC5 & 78 & 11 & 0.941 & 0.904 \\
\hline SAC6 & 297 & 16 & 1 & 0.833 \\
\hline SAC7 & 20 & 5 & 0.642 & 0.919 \\
\hline SAC10 & 72 & 13 & 0.983 & 0.885 \\
\hline SAC11 & 171 & 14 & 0.994 & 0.867 \\
\hline SAC14 & 21 & 10 & 0.886 & 0.886 \\
\hline SAC18 & 46 & 9 & 0.858 & 0.9 \\
\hline SAC20 & 27 & 7 & 0.781 & 0.925 \\
\hline SAC21 & 16 & 4 & 0.545 & 0.9 \\
\hline SAC23 & 24 & 10 & 0.924 & 0.924 \\
\hline SAC24 & 85 & 12 & 0,984 & 0.911 \\
\hline SAC25 & 44 & 14 & 0.967 & 0.843 \\
\hline SAC26 & 121 & 14 & 0.898 & 0.783 \\
\hline SAC27 & 59 & 10 & 0.945 & 0.945 \\
\hline SAC28 & 28 & 10 & 0.899 & 0.877 \\
\hline SAC29 & 60 & 12 & 1 & 0.925 \\
\hline SAC31 & 14 & 11 & 1 & 0.877 \\
\hline Referen
\end{tabular}

Referencias: n: tamaño de la muestra, k: cantidad de categorías, $\mathrm{H}$ : índice de riqueza, J: índice de homogeneidad.

concentraciones que presentan valores intermedios (SAC 7, SAC 20 y SAC 21). Al mismo tiempo, los valores altos y muy similares de $\mathrm{J}$ en los conjuntos dan cuenta de una distribución equitativa de los distintos tipos de artefactos formatizados en todas las concentraciones. La excepción puede ser la concentración SAC 26 ya que presenta el valor de J más bajo.

Por otro lado, debe tenerse en cuenta que la diversidad de un conjunto puede estar en relación directa con el tamaño de la muestra y no así con los comportamientos desarrollados (Jones et al. 1983; Meltzer et al. 1992; Shott 1989). Por eso se considera relevante evaluar en qué medida la diversidad de cada uno de los conjuntos está en relación con el tamaño de la muestra de los mismos.

Como se observa en figura 2 , a medida que aumenta el tamaño de la muestra de los conjuntos aumenta la cantidad de clases artefactuales representadas. En este sentido, el coeficiente de correlación entre ambas variables es de 0.672 , señalando la existencia de una relación media entre las mismas. Sin embargo, el coeficiente de determinación $\left(\mathrm{r}^{2}=\right.$ 0.451) indica que el tamaño de la muestra explica en un $45.1 \%$ la variabilidad en la cantidad de clases 


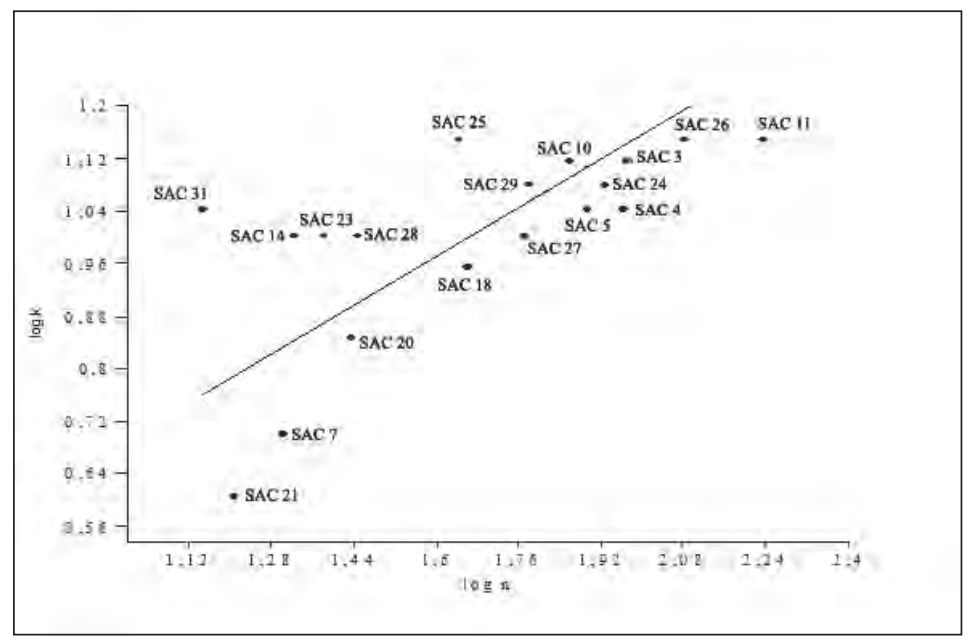

Fig. 2. Riqueza artefactual y tamaño de la muestra r: 0,67 p: 0,002.

Referencias: log.: logaritmo, n: tamaño de la muestra, k: cantidad de categorías.

representadas. Tanto en el gráfico como en el cálculo de esta correlación no fue incluida la muestra de la concentración de SAC 6 ya que se comporta como un outlier, con un tamaño de muestra muy superior al de las otras concentraciones.

Al mismo tiempo, como la relación entre el tamaño de la muestra y la cantidad de clases artefactuales es parcial, se observa que los conjuntos tienden a agruparse de dos maneras diferentes. Por un lado, un conjunto de las concentraciones artefactuales (SAC 3, SAC 4, SAC 5, SAC 10, SAC 11, SAC 24, SAC 25, SAC 26 y SAC 29) presenta tamaños de la muestra diferentes pero la cantidad de clases artefactuales varía entre 11 y 14 . Por otro lado, un segundo grupo (SAC 7, SAC 14, SAC 20 SAC 21, SAC 23 y SAC 28) se caracteriza por presentar tamaños de la muestra similares (no superan los 30 artefactos) pero con una gran variación en la cantidad de clases representadas (entre 4 y 10 clases). Esto especifica aún más las diferencias entre los conjuntos en cuanto a la diversidad artefactual.

Por otra parte, también se evaluó la diversidad en el material de las prospecciones y transectas (tabla 6). Se debe tener en cuenta que, a diferencia del material de las concentraciones artefactuales, el relevamiento in situ de las clases artefactuales en las transectas por distintas personas puede afectar en alguna medida los valores de los índices considerados.

En este caso se observa que los valores de riqueza tienden a ser variables entre las prospecciones y transectas y a ser menores que los de las concentraciones artefactuales. Los relevamientos que presentan valores mayores son los que están vinculados con las concentraciones artefactuales de SAC 3, SAC 5 y el sector de entierros de SAC 1. Al mismo tiempo, los valores altos de $\mathrm{J}$ en los conjuntos dan cuenta de una distribución equitativa

Tabla 6. Riqueza y homogeneidad artefactual prospecciones y transectas.

\begin{tabular}{|c|c|c|c|c|}
\hline & $\mathbf{n}$ & $\mathbf{k}$ & $\mathbf{H}$ & $\mathbf{J}$ \\
\hline SAC1 BARDA ESTE & 6 & 2 & 0,195 & 0,65 \\
\hline SAC1 BARDA OSTE & 14 & 8 & 0,839 & 0,932 \\
\hline SAC2 CH8 & 15 & 6 & 0,702 & 0,911 \\
\hline SAC2/5-SAC30 A & 1 & 1 & 0 & - \\
\hline SAC2/5-SAC30 B & 2 & 2 & 0,301 & 1 \\
\hline SAC3 TR 250 & 12 & 4 & 0,537 & 0,895 \\
\hline SAC3 TR 50 & 10 & 4 & 0,46 & 0,766 \\
\hline SAC3 TR 130 ${ }^{\circ}$ & 46 & 13 & 0,939 & 0,845 \\
\hline SAC3 TR 310 & 9 & 4 & 0,552 & 0,87 \\
\hline SAC3 TR 350 & 4 & 3 & 0,451 & 0,959 \\
\hline SAC5 TR 2 & 5 & 4 & 0,578 & 0,963 \\
\hline SAC5 TR 3 & 5 & 5 & 0,698 & 1 \\
\hline SAC5 TR 4 & 8 & 4 & 0,466 & 0,776 \\
\hline SAC5 TR 5 & 11 & 6 & 0,747 & 0,97 \\
\hline SAC29-COTA600 & 3 & 3 & 0,477 & 1 \\
\hline SAC1-SAC14 & 8 & 3 & 0,423 & 0,9 \\
\hline CHACRA-SAC31 & 2 & 1 & 0 & - \\
\hline CASCO-SAC4 & 2 & 2 & 0,301 & 1 \\
\hline SAC10-RIO & 2 & 2 & 0,301 & 1 \\
\hline RIO-CAMINO & 2 & 2 & 0,301 & 1 \\
\hline
\end{tabular}

Referencias: n: tamaño de la muestra, k: cantidad de categorías, H: índice de riqueza, J: índice de homogeneidad. 
Tabla 7. Artefactos formatizados representados en mayor frecuencia.

\begin{tabular}{|c|c|c|c|c|c|c|c|}
\cline { 2 - 7 } \multicolumn{1}{c|}{} & afs & nuc & pta & rd & rp & $\%$ & n \\
\hline SAC3 & $\mathbf{3 1 , 3 7}$ & 17,65 & 27,45 & 9,8 & 13,73 & 100 & 51 \\
\hline SAC4 & 21,57 & $\mathbf{3 9 , 2 2}$ & 3,92 & 9,8 & 25,49 & 100 & 51 \\
\hline SAC5 & 11,32 & 22,64 & 26,42 & 9,43 & $\mathbf{3 0 , 1 9}$ & 100 & 53 \\
\hline SAC6 & 7,01 & 18,47 & 10,19 & 17,83 & $\mathbf{4 6 , 5}$ & 100 & 157 \\
\hline SAC7 & - & - & 25 & $\mathbf{3 7 , 5}$ & $\mathbf{3 7 , 5}$ & 100 & 16 \\
\hline SAC10 & 10,26 & 20,51 & 17,95 & 7,69 & $\mathbf{4 3 , 5 9}$ & 100 & 39 \\
\hline SAC11 & $\mathbf{2 9 , 1 3}$ & 19,42 & 12,62 & 13,59 & 25,24 & 100 & 103 \\
\hline SAC14 & 21.42 & $\mathbf{5 0}$ & - & 14.28 & 14.28 & 100 & 14 \\
\hline SAC18 & 16.67 & 13.89 & 11.11 & 19.44 & $\mathbf{3 8 . 8 9}$ & 100 & 36 \\
\hline SAC20 & 20 & 30 & - & 10 & $\mathbf{4 0}$ & 100 & 20 \\
\hline SAC21 & - & 12,5 & $\mathbf{3 7 , 5}$ & 12,5 & $\mathbf{3 7 , 5}$ & 100 & 16 \\
\hline SAC23 & 22,22 & $\mathbf{2 7 , 7 8}$ & 11,11 & 16,67 & 22,22 & 100 & 18 \\
\hline SAC24 & 22,64 & 20,75 & 16,98 & 7,55 & $\mathbf{3 2 , 0 8}$ & 100 & 53 \\
\hline SAC25 & 4 & 4 & $\mathbf{4 4}$ & 36 & 12 & 100 & 25 \\
\hline SAC26 & 8,6 & 19,35 & 13,98 & 12,9 & $\mathbf{4 5 , 1 6}$ & 100 & 93 \\
\hline SAC27 & 21,05 & 15.78 & 7.9 & $\mathbf{3 1 . 5 7}$ & 23.68 & 100 & 38 \\
\hline SAC28 & 17.64 & 35.3 & - & - & $\mathbf{4 7 . 0 5}$ & 100 & 17 \\
\hline SAC29 & 15,79 & 23,68 & 10,53 & 18,42 & $\mathbf{3 1 , 5}$ & 100 & 38 \\
\hline SAC31 & 25 & 25 & 25 & 25 & - & 100 & 4 \\
\hline total & 15.9 & 20.78 & 14.6 & 14.9 & $\mathbf{3 3 . 6}$ & 100 & 842 \\
\hline
\end{tabular}

Referencias: afs: artefactos de formatización sumaria, nuc, núcleos, pta: puntas de proyectil, rd: raederas, rp: raspadores.

de los distintos tipos de artefactos formatizados en todos los sectores. Es de resaltar que estos valores tienden a ser superiores a los registrados en las concentraciones artefactuales.

Por otro lado, el coeficiente de correlación entre el tamaño de la muestra y la cantidad de clases artefactuales es de 0,927 , con un coeficiente de determinación de 0,856. Lo cual indica que el tamaño de la muestra explica en un 85,6\% la variabilidad en la cantidad de clases representadas. Es así que, en las prospecciones y transectas a medida que aumenta el tamaño de la muestra de los conjuntos aumenta la cantidad de clases artefactuales representadas.

Tipos de artefactos formatizados

La información referida a los tipos de artefactos formatizados fue presentada en la sección anterior (tabla 3). Se debe aclarar que, en este caso, para el análisis de la estructura artefactual de los conjuntos, se incluyen los núcleos, nódulos y los filos naturales con rastros complementarios a pesar de no ser artefactos formatizados.

Como se ha mencionado previamente, existe una alta representación de raspadores y núcleos en casi todos los conjuntos del área. Con porcentajes variables le siguen en importancia los fragmentos de artefactos formatizados, los artefactos de formatización sumaria, las raederas y las puntas de proyectil (ver tabla 3).

Para observar cómo se distribuyen estas clases artefactuales en los diferentes conjuntos, en la siguiente tabla se presentan sus porcentajes en relación al total de artefactos más representados (tabla 7). En este análisis no fueron considerados los fragmentos de artefactos formatizados dado el carácter indiferenciado de los mismos.

Se observa variabilidad en los conjuntos con respecto a los tipos de artefactos formatizados más representados. Así, los raspadores predominan en la mayoría de los conjuntos (SAC 5, SAC 6, SAC 7, SAC 10, SAC 18, SAC 21, SAC 24, SAC 26, SAC 28 y SAC 29). Los núcleos se encuentran más representados en SAC 4, SAC 14 y SAC 23. Los artefactos de formatización sumaria tienen una mayor frecuencia en SAC 3 y SAC 11. Por su parte, las puntas de proyectil se presentan con porcentajes altos en SAC 21 y SAC 25 y las raederas en SAC 7, SAC 25 y SAC 27. En el conjunto artefactual de SAC 31, exceptuando la ausencia de raederas, los distintos tipos de artefactos formatizados están en 


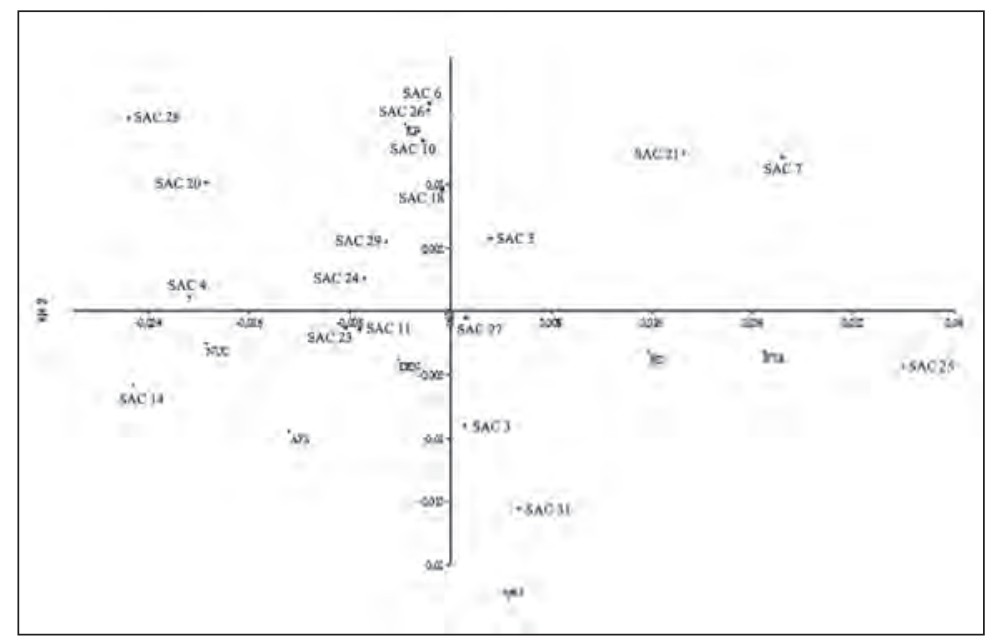

Fig. 3. Análisis de correspondencia.

los mismos porcentajes. De esta manera, al igual que lo especificado con respecto a los índices de riqueza artefactual y la estructura artefactual general de las concentraciones, de este análisis se desprenden diferencias entre las mismas.

Para establecer posibles agrupamientos entre los diferentes conjuntos, en la siguiente figura se presenta la misma información estandarizada junto con los correspondientes valores de riqueza y densidad artefactual pero en términos del análisis de correspondencia (figura 3).

Por un lado, se observa que en el eje 1 , que explica el 46, 1\% de la variabilidad, se oponen los raspadores y los artefactos de formatización sumeria. Por otro lado, en el eje 2, que explica el 22, 7\% de la variabilidad, se encuentran opuestos las puntas de proyectil y las raederas a los núcleos. Esto permite dar cuenta de la existencia de un agrupamiento de las concentraciones de SAC 5, SAC 6, SAC 10, SAC 11, SAC 24, SAC 27, SAC 29 que se caracterizan, en mayor o menor medida, por un predominio de los raspadores y proporciones similares del resto de las clases artefactuales. La concentración SAC 3 tiene algunas semejanzas con este conjunto pero tiene una mayor frecuencia de artefactos de formatización sumaria. Otro agrupamiento lo constituyen las concentraciones de SAC 4, SAC 14 y SAC 20 que presentan valores similares de núcleos y artefactos de formatización sumaria y la ausencia de puntas de proyectil. En menor grado, el conjunto de SAC 28 presenta algunas similitudes con este grupo. Por otro lado, las muestras de SAC 7, SAC 21 y SAC 25 conforman un conjunto de concentraciones artefactuales donde las puntas de proyectil y las raederas tienen valores mayores.

En términos generales, se registra un gran número de concentraciones con una alta diversidad artefactual, donde dominan los raspadores, $y$ concentraciones con una menor diversidad, con frecuencias mayores de puntas de proyectil, raederas o núcleos. Entre estas concentraciones también se han observado contrastes, vinculados con la representación diferencial de los artefactos de molienda y la cerámica. También deben incluirse las diferencias observadas en el material proveniente de las prospecciones y transectas. En este caso, si no se consideran los relevamientos próximos a concentraciones importantes de material, como SAC 3 y SAC 5, la densidad y riqueza artefactual es baja y se destaca la frecuencia de núcleos y bifaces.

Estas discrepancias apuntan a la existencia de variabilidad en el uso de los espacios ubicados en el fondo de cuenca. Así, puede pensarse que espacios, principalmente médanos vegetados con molles, podrían estar funcionando a modo de campamentos donde se desarrollarían una amplia variedad de tareas relacionadas con el procesamiento y consumo de recursos y producción de tecnofacturas. A su vez, existirían espacios donde se efectuarían actividades más específicas, quizás vinculadas con la obtención de recursos. 
Tabla 8. Tipos de desechos de talla enteros y fracturados con talón.

\begin{tabular}{|c|c|c|c|c|c|c|c|c|c|c|c|c|c|c|c|c|}
\hline & ls.r.n. & ls.r.ins. & ls.ab & ls.pl & ls.ar & ls.an & ls.in & indi & ls.nod & ls.se & ls.pr & ls.dn & lam & hj & \% & n \\
\hline SAC3 & 0.2 & 3.9 & - & 4.14 & 10.45 & $\mathbf{5 6 . 2}$ & 1.9 & - & - & 8.3 & 6.31 & 6.31 & 0.65 & 1.52 & 100 & 459 \\
\hline SAC4 & 2.12 & - & - & 5.67 & 6.4 & $\mathbf{5 4 . 6}$ & 12.05 & & - & 2.83 & 5.67 & 3.54 & 0.7 & 6.4 & 100 & 141 \\
\hline SAC5 & 2.32 & 1.66 & 2.32 & 3.32 & 7.3 & $\mathbf{5 7 . 5}$ & 0.66 & 0.33 & - & 4.32 & 9.3 & 9.06 & 0.66 & 0.33 & 100 & 301 \\
\hline SAC6 & 4.05 & - & 2.7 & 2.7 & 6.75 & 43.2 & 12.2 & - & - & 10.8 & 8.12 & 9.45 & - & - & 100 & 74 \\
\hline SAC7 & 4.76 & - & - & - & 9.52 & 42.86 & - & - & - & 23.8 & 4.76 & 4.76 & - & 9.52 & 100 & 21 \\
\hline SAC10 & 5.26 & 3.15 & - & 7.36 & 13.7 & 38.9 & 6.3 & - & - & 6.3 & 8.42 & 8.42 & - & 2.1 & 100 & 95 \\
\hline SAC11 & 1.9 & 0.7 & 0.17 & 7.3 & 13.5 & $\mathbf{5 5 . 8}$ & 1.9 & - & 0.17 & 5.2 & 5.56 & 6.26 & 0.34 & 1.04 & 100 & 575 \\
\hline SAC14 & 7.14 & - & - & - & 25 & 25 & 7.14 & - & - & 7.14 & 7.14 & 17.85 & - & 3.57 & 100 & 28 \\
\hline SAC18 & 3.54 & - & - & 7.08 & 15.9 & $\mathbf{5 1 . 3}$ & 4.4 & 0.88 & - & 6.2 & 3.54 & 4.4 & - & 2.6 & 100 & 113 \\
\hline SAC20 & 3.06 & - & - & 3.06 & 28.57 & 38.7 & 1.02 & - & - & 9.18 & 3.06 & 9.18 & 2.04 & 2.04 & 100 & 98 \\
\hline SAC21 & - & - & - & 2.56 & 10.25 & $\mathbf{5 8 . 9}$ & - & - & - & 2.56 & 10.25 & 7.7 & 2.56 & 5.13 & 100 & 39 \\
\hline SAC23 & - & - & 2.27 & 2.27 & 9.09 & $\mathbf{5 9 . 1}$ & - & - & - & 9.09 & 4.5 & 13.6 & - & - & 100 & 44 \\
\hline SAC24 & 1.97 & 0.45 & 0.3 & 6.35 & 15.58 & $\mathbf{5 2 . 0 4}$ & 1.66 & 2.12 & - & 4.54 & 6.66 & 7.72 & 0.45 & 0.15 & 100 & 661 \\
\hline SAC25 & 1.34 & 0.67 & 2.01 & 6.04 & 14.1 & $\mathbf{6 3 . 0 8}$ & 0.67 & - & - & 4.69 & 2.68 & 2.68 & - & 2.01 & 100 & 149 \\
\hline SAC26 & 3.06 & 3.06 & 8.16 & 3.06 & 9.2 & 43.8 & - & - & - & 8.16 & 6.12 & 14.28 & 1.02 & & 100 & 98 \\
\hline SAC27 & - & - & - & 3.27 & 9.8 & $\mathbf{6 0 . 6}$ & 9.8 & - & - & 8.2 & 3.27 & 3.27 & - & 1.6 & 100 & 61 \\
\hline SAC28 & - & 2.9 & - & - & 17.6 & 47.0 .5 & 5.8 & - & - & 5.8 & 5.8 & 8.8 & - & 5.8 & 100 & 34 \\
\hline SAC29 & 2.4 & 2.4 & 2.4 & 2.4 & 10.8 & $\mathbf{5 1 . 8}$ & 1.2 & - & - & 6.02 & 2.4 & 10.8 & 2.4 & 4.8 & 100 & 83 \\
\hline SAC31 & 5.26 & - & - & - & 5.26 & 31.57 & 5.26 & - & 5.26 & 5.26 & 5.26 & 36.8 & - & - & 100 & 19 \\
\hline Total & 1,9 & 1,2 & 0.84 & 5,14 & 12.7 & 53.1 & 2,72 & 0.52 & 0,06 & 5,98 & 6,08 & 7.57 & 0,5 & 1.5 & 100 & 3093 \\
\hline
\end{tabular}

Referencias: hj: hojas, indi: indiferenciado, lam: lámina, ls.an: lasca angular, ls.ar: lasca de arista, ls.pl: lasca plana,

ls.nod: lasca nodular, ls.dn: lasca de dorso natural, ls.pr: lasca primaria, ls.sec: lasca secundaria, ls.r.n.: lasca de reactivación de núcleo, ls.ab: lasca de adelgazamiento bifacial, ls.r.ins: lasca de reactivación de instrumentos.

Tipos de desechos de talla

Para especificar aún más las características de la estructura artefactual de la cuenca, la tabla 8 presenta las frecuencias de los diferentes tipos de desechos de talla, que se encuentran enteros y fracturados con talón, registrados en las concentraciones artefactuales. Para este análisis se tuvo en cuenta las consideraciones metodológicas realizadas por Bellelli y colaboradores (1985-1987).

En primer lugar, se observa una gran variedad de tipos de desechos representados en el conjunto lítico general, que incluye no sólo diferentes clases de lascas externas (19,7 \%) e internas (70,9\%) sino también lascas de reactivación de instrumentos (lascas de reactivación directa e inversa), de reactivación de núcleos (lascas tableta y flanco de núcleo) y hojas, entre otros. En principio, esta variedad podría estar dando cuenta de la representación de las distintas etapas de manufactura y reactivación de artefactos formatizados.

Al mismo tiempo, existe una alta frecuencia de las lascas internas, principalmente lascas angulares y de arista en la mayoría de las concentraciones artefactuales. Las lascas externas (lascas primarias, secundarias y de dorso natural) le siguen en importancia, fundamentalmente en concentraciones como SAC 7, SAC 14 y SAC 31. A su vez, es de destacar la presencia de lascas de reactivación de núcleos en casi todos los conjuntos. Por último, cabe mencionar la representación de hojas en el sector de SAC 4, tendencia registrada en las formas base de los artefactos formatizados (Cassiodoro 2008).

Para un estudio más detallado de la representación de las distintas etapas de manufactura de artefactos formatizados se requiere del análisis de variables como los tamaños de los desechos de talla y las materias primas utilizadas, por cuestiones de espacio esta información no se presenta en este trabajo.

\section{Materias primas}

Teniendo en cuenta los objetivos de este trabajo, un aspecto más para caracterizar la composición de los conjuntos tecnológicos son las ma- 
Tabla 9. Materia primas lítica en concentraciones artefactuales.

\begin{tabular}{|c|c|c|c|c|c|c|c|c|c|c|c|c|c|c|}
\hline & \multicolumn{2}{|c|}{ basalto } & \multicolumn{2}{c|}{ limolita } & \multicolumn{2}{c|}{ obsidiana } & \multicolumn{2}{c|}{ otras } & \multicolumn{2}{|c|}{ riolita } & \multicolumn{2}{c|}{ sílice } & \multicolumn{2}{c|}{ total } \\
\cline { 2 - 18 } & $\mathrm{n}$ & $\%$ & $\mathrm{n}$ & $\%$ & $\mathrm{n}$ & $\%$ & $\mathrm{n}$ & $\%$ & $\mathrm{n}$ & $\%$ & $\mathrm{n}$ & $\%$ & $\mathrm{n}$ & $\%$ \\
\hline SAC3 & 27 & 3.37 & 2 & 0.25 & 641 & $\mathbf{7 9 . 2 3}$ & 4 & 0.49 & 29 & 3.58 & 106 & 13.1 & 809 & 100 \\
\hline SAC4 & 152 & $\mathbf{4 1 . 8 7}$ & - & - & 81 & 22.31 & 16 & 4.41 & 26 & 7.16 & 88 & 24.24 & 363 & 100 \\
\hline SAC5 & 40 & 7.64 & 1 & 0.19 & 316 & $\mathbf{6 0 . 4 2}$ & 16 & 3.06 & 9 & 1.72 & 141 & 26.96 & 523 & 100 \\
\hline SAC6 & 74 & 17.05 & - & - & 166 & $\mathbf{3 8 . 2 5}$ & 54 & 12.44 & 15 & 3.46 & 125 & 28.8 & 434 & 100 \\
\hline SAC7 & 9 & 19.57 & 2 & 4.35 & 17 & $\mathbf{3 6 . 9 6}$ & - & - & 3 & 6.52 & 15 & 32.61 & 46 & 100 \\
\hline SAC10 & 46 & 19.82 & - & - & 71 & 30.6 & 18 & 7.75 & 11 & 4.74 & 86 & $\mathbf{3 7 . 0 7}$ & 232 & 100 \\
\hline SAC11 & 370 & $\mathbf{3 4 , 6 7}$ & 11 & 1,03 & 192 & 17,99 & 65 & 6,09 & 97 & 9,09 & 332 & 31,11 & 1067 & 100 \\
\hline SAC14 & 24 & $\mathbf{3 5 . 8 2}$ & 1 & 1,49 & 16 & 23.88 & 6 & 8.95 & 2 & 2.98 & 18 & 26.86 & 67 & 100 \\
\hline SAC18 & 91 & 34.6 & 3 & 1.14 & 105 & $\mathbf{3 9 . 9}$ & 5 & 1.9 & 7 & 2.66 & 52 & 19.8 & 263 & 100 \\
\hline SAC20 & 53 & 29.44 & - & - & 59 & $\mathbf{3 2 . 7 7}$ & 6 & 3.33 & 9 & 5 & 53 & 29.44 & 180 & 100 \\
\hline SAC21 & 8 & 10.81 & - & - & 49 & $\mathbf{6 6 . 2 1}$ & 1 & 1.35 & 1 & 1.35 & 15 & 20.27 & 74 & 100 \\
\hline SAC23 & 7 & 7.45 & - & - & 44 & $\mathbf{4 6 . 8 1}$ & 6 & 6.38 & 13 & 13.83 & 24 & 25.53 & 94 & 100 \\
\hline SAC24 & 319 & 15.5 & 11 & 0.5 & 1421 & $\mathbf{6 9 . 0 8}$ & 9 & 0.43 & 28 & 1.36 & 269 & 13.07 & 2057 & 100 \\
\hline SAC25 & 41 & 14,29 & - & - & 118 & $\mathbf{4 1 , 1 1}$ & 13 & 4,53 & 1 & 0.35 & 114 & 39,72 & 287 & 100 \\
\hline SAC26 & 51 & 18,89 & 4 & 1.48 & 133 & $\mathbf{4 9 , 2 6}$ & 9 & 3,33 & 6 & 2.34 & 67 & 24,81 & 270 & 100 \\
\hline SAC27 & 77 & $\mathbf{4 5 . 5 6}$ & - & - & 37 & 21.89 & 4 & 2.36 & 1 & 0.59 & 50 & 29.58 & 169 & 100 \\
\hline SAC28 & 10 & 12.19 & 3 & 3.65 & 28 & $\mathbf{3 4 . 1 4}$ & 8 & 9.75 & 5 & 6.09 & 28 & $\mathbf{3 4 . 1 4}$ & 82 & 100 \\
\hline SAC29 & 65 & 30.23 & - & - & 80 & $\mathbf{3 7 . 2}$ & 17 & 7.9 & 4 & 1.86 & 49 & 22.79 & 215 & 100 \\
\hline SAC33 & 11 & 20.37 & - & - & 33 & $\mathbf{6 1 . 1 1}$ & 5 & 9.25 & 2 & 3.7 & 3 & 5.55 & 54 & 100 \\
\hline total & 1475 & 20.2 & 38 & 0.52 & 3607 & $\mathbf{4 9 . 5}$ & 262 & 3.6 & 269 & 3.7 & 1635 & 22.4 & 7286 & 100 \\
\hline
\end{tabular}

terias primas utilizadas para la manufactura de los diferentes artefactos. En la cuenca se ha registrado la disponibilidad de materias primas líticas y arcillas. Las primeras incluyen la andesita o basalto Posadas y una serie de otras materias primas de variada calidad y frecuencias (rocas silíceas, riolita, limolita, etc.) (Guraieb 1998). También están disponibles guijarros y bloques utilizables para la manufactura de artefactos de molienda y la construcción de estructuras, respectivamente (Cassiodoro 2005). Por su parte, la existencia de distintos depósitos secundarios de arcillas vinculados con ambientes donde existen sedimentos de origen aluvial y lacustre en la cuenca hace referencia a la disponibilidad local de materias primas cerámicas. A partir del estudio de cortes delgados se ha registrado variabilidad en la composición mineralógica de los sedimentos y arcillas del área (Cassiodoro y Tchilinguirian 2007). La materia prima que no se encuentra en la cuenca es la obsidiana negra, la cual está disponible en Pampa del Asador. En este sector, distante unos $45 \mathrm{~km}$ del área, también existen guijarros de rocas silíceas y basalto de muy buena calidad (Espinosa y Goñi 1999).
En la siguiente tabla se presenta la frecuencia de los tipos de materias primas representadas en la producción lítica total de los conjuntos de las concentraciones artefactuales (tabla 9).

En las concentraciones artefactuales de la cuenca del lago Salitroso la obsidiana es la materia prima lítica que se encuentra en más frecuencia (tabla 9). La misma es seguida en importancia por las rocas silíceas y la andesita o basalto Posadas. A su vez, se observa que existen diferencias entre las concentraciones con respecto a las materias primas líticas utilizadas ya que en algunas de ellas hay una mayor representación del basalto (SAC 4, SAC 14, SAC 27 y SAC 11) y en otras de las rocas silíceas (SAC 10).

Por su parte, en las prospecciones y transectas, la obsidiana y las rocas silíceas están representadas en porcentajes similares (tabla 10). Al mismo tiempo, se observa la existencia de sectores, como las transectas próximas a SAC 25 y la prospección entre SAC 1 y SAC 14, donde predomina el basalto. 
Tabla 10. Materias primas líticas de prospecciones y transectas.

\begin{tabular}{|c|c|c|c|c|c|c|c|c|c|c|c|c|c|c|}
\hline & \multicolumn{2}{|c|}{ basalto } & \multicolumn{2}{|c|}{ limolita } & \multicolumn{2}{|c|}{ obsidiana } & \multicolumn{2}{|c|}{ otras } & \multicolumn{2}{|c|}{ riolita } & \multicolumn{2}{|c|}{ sílice } & \multicolumn{2}{|c|}{ total } \\
\hline & $\mathrm{n}$ & $\%$ & $\mathrm{n}$ & $\%$ & $\mathrm{n}$ & $\%$ & $\mathrm{n}$ & $\%$ & $\mathrm{n}$ & $\%$ & $\mathrm{n}$ & $\%$ & $\mathrm{n}$ & $\%$ \\
\hline SAC1 BARDA ESTE & 1 & 9.1 & - & - & 5 & 45.45 & - & - & - & - & 5 & 45.45 & 11 & 100 \\
\hline SAC1 BARDA OEST & - & - & - & - & 30 & 20.13 & 1 & 0.67 & - & - & 118 & 79.19 & 149 & 100 \\
\hline SAC2 CH8 & 8 & 16.32 & - & - & 22 & 44.89 & 1 & 2.04 & 1 & 2.04 & 17 & 34.69 & 49 & 100 \\
\hline SAC2/5-SAC30 A & 7 & 33.33 & - & - & 12 & 57.14 & - & - & - & - & 2 & 9.52 & 21 & 100 \\
\hline SAC2/5-SAC30 B & 1 & 14.28 & - & - & 3 & 42.85 & 1 & 14.28 & - & - & 2 & 28.57 & 7 & 100 \\
\hline SAC3 TR $250^{\circ}$ & - & - & - & - & - & - & 10 & 66.66 & - & - & 5 & 33.33 & 15 & 100 \\
\hline SAC3 TR $50^{\circ}$ & 2 & 18.18 & - & - & 3 & 27.27 & 6 & 54.54 & - & - & - & - & 11 & 100 \\
\hline SAC3 TR $130^{\circ}$ & 34 & 15.31 & 1 & 0.45 & 105 & 47.29 & 17 & 7.65 & 2 & 0.9 & 63 & 28.37 & 222 & 100 \\
\hline SAC3 TR $310^{\circ}$ & - & - & - & - & 2 & 20 & - & - & 2 & 20 & 6 & 60 & 10 & 100 \\
\hline SAC3 TR 350응 & - & - & - & - & 3 & 50 & - & - & 2 & 33.33 & 1 & 16.6 & 6 & 100 \\
\hline SAC5 TR1 & 1 & 100 & - & - & - & - & - & - & - & - & - & - & 1 & 100 \\
\hline SAC5 TR2 & - & - & - & - & 4 & 66.66 & - & - & - & - & 2 & 33.33 & 6 & 100 \\
\hline SAC5 TR3 & 1 & 9.1 & - & - & 1 & 9.1 & 3 & 27.2 & 2 & 18.2 & 4 & \begin{tabular}{|l|}
36.4 \\
\end{tabular} & 11 & 100 \\
\hline SAC5 TR4 & 7 & 16.66 & - & - & 20 & 47.62 & 6 & 14.28 & 1 & 2.38 & 8 & 19.04 & 42 & 100 \\
\hline SAC5 TR5 & - & - & 2 & 10.52 & 7 & 36.84 & - & - & 4 & 21.05 & 6 & 31.58 & 19 & 100 \\
\hline SAC25 C2 TR 1 & 12 & 70.58 & - & - & 1 & 5.88 & 1 & 5.88 & 2 & \begin{tabular}{|l|}
11.76 \\
\end{tabular} & 1 & 5.88 & 17 & 100 \\
\hline SAC25 C2 TR 2 & 4 & 100 & - & - & - & - & - & - & - & - & - & - & 4 & 100 \\
\hline SAC29-COTA600 & 1 & 14.28 & - & - & 4 & 57.14 & - & - & - & - & 2 & 28.57 & 7 & 100 \\
\hline SAC1-SAC14 & 14 & 51.85 & - & - & 9 & 33.33 & - & - & 3 & \begin{tabular}{|l|}
11.11 \\
\end{tabular} & 1 & 3.7 & 27 & 100 \\
\hline CHACRA-SAC33 & 2 & 13.33 & - & - & 10 & 66.66 & 2 & 13.33 & - & - & 1 & 6.66 & 15 & 100 \\
\hline CASCO-SAC4 & - & - & - & - & 4 & 100 & - & - & - & - & - & - & 4 & 100 \\
\hline SAC10-RIO & 1 & 16.66 & - & - & 4 & 66.66 & 1 & 16.66 & - & - & - & - & 6 & 100 \\
\hline RIO-CAMINO & - & - & - & - & 4 & 100 & - & - & - & - & - & - & 4 & 100 \\
\hline total & 96 & 14.45 & 3 & 0.45 & 253 & 38.1 & 49 & 7.38 & 19 & 2.86 & 244 & \begin{tabular}{|l|}
36.74 \\
\end{tabular} & 664 & 100 \\
\hline
\end{tabular}

\section{DISCUSIÓN}

En un contexto de movilidad residencial reducida, se ha propuesto que la cuenca del lago Salitroso constituye un espacio óptimo para fines residenciales debido a que presenta características de un semidesierto de baja altura sobre el nivel del mar con disponibilidad de diferentes recursos. El modelo de poblamiento tardío sostiene que, a nivel regional, las condiciones ambientales y las características de la estructura de recursos de esta cuenca favorecen mejor el asentamiento prolongado que otros espacios (Goñi 2000). Por lo tanto, se propone que las características de los conjuntos tecnológicos estarían condicionadas por el carácter residencial y la mayor duración de las ocupaciones en esta cuenca lacustre baja.

Una primera expectativa planteada con respecto al uso residencial de la cuenca considera que todos los sectores deberían haber sido frecuentemente utilizados, por lo cual el registro tecnológico se distribuirá de manera continua y densa. En vinculación con esta expectativa, se ha establecido la distribución continua de material arqueológico en todos los sectores. Sin embargo, el registro arqueológico se distribuye diferencialmente en el espacio en relación a determinadas geoformas. Por un lado, existe un gran número de concentraciones artefactuales de superficie en médanos fijados por vegetación, con variada densidad, separadas entre sí por espacios donde la densidad artefactual es muy baja. Por otro lado, al carácter puntual de las mismas, se le suman las diferentes estructuras de entierros humanos localizadas en lugares específicos del paisaje (principalmente terrazas, cerros bajos y bardas). Características similares de la distribución del registro arqueológico han sido evidenciadas en las inmediaciones de los lagos Posadas y Pueyrredón (Re 2006). Esta distribución no responde a problemas de visibilidad del registro arqueológico.

A su vez, existe una distribución diferencial de las tecnologías en los distintos espacios. La cerámica y los artefactos de molienda tienden a registrarse en los sectores de médanos con mayor 
densidad artefactual, mientras que los entierros en estructuras de piedra tienden a registrarse en sectores de terrazas, cerros de escasa altura y bardas. Asociado a estas estructuras hay una baja frecuencia de artefactos en general, estando los artefactos de metal, las cuentas de valva y vidrio exclusivamente en relación con ellas (Cassiodoro y García Guráieb 2009). En estas estructuras no están presentes raspadores y artefactos de formatización sumaria, los cuales están representados en alta frecuencia en las concentraciones artefactuales en médanos.

Por otra parte, cabe mencionar que en el área existe una serie de evidencias que se vinculan con la redundancia de uso de los espacios. En primer lugar, se encuentran las distintas fechas asignables al Holoceno tardío disponibles para Cerro de los Indios 1 (De Nigris et al. 2004), distante unos $20 \mathrm{~km}$ de la cuenca en estudio, y las cronologías exclusivamente tardías (2.600-300 años AP) de la cuenca del lago Salitroso y de las concentraciones a cielo abierto de la cuenca del lago Posadas (Goñi et al. 2000-2002). A su vez, debe considerarse la densidad artefactual en la cuenca del lago Salitroso, dada no sólo por una alta representación de artefactos sino también por estructuras de piedra en un espacio relativamente acotado. Por otro lado, existen evidencias de redundancia en el uso de determinados puntos del espacio, más allá de la posibilidad de palimpsesto, como por ejemplo estructuras de entierro con reutilización en diferentes momentos cronológicos, concentraciones artefactuales con variabilidad de morfologías de puntas de proyectil, existencia de más de un recipiente cerámico en las concentraciones de SAC 3 y SAC 5 , presencia de pátinas diferenciales en artefactos líticos (Goñi y Barrientos 2000; Cassiodoro 2008).

Asimismo, en el marco de una movilidad residencial reducida y una consecuente ocupación permanente o semi-permanente de la cuenca se ha planteado, como otra expectativa, la existencia del equipamiento de los espacios con elementos tecnológicos que presentan diseños poco transportables o no transportables. Esto ha sido discutido específicamente en trabajos anteriores (Cassiodoro 2005), no obstante cabe mencionar que el registro arqueológico se caracteriza por:

-Un 68\% de las concentraciones artefactuales de la cuenca contienen artefactos de molienda.

-Se han registrado rocas sin formatización de dimensiones similares a los artefactos de molienda que fueron transportadas a varias de las concentraciones artefactuales.

-Las estructuras de piedra, principalmente chenques registrados en alta frecuencia en la cuenca, también estarían equipando espacios.

De esta manera, la recurrencia de artefactos con diseños poco transportables en las concentraciones artefactuales de superficie localizadas en médanos estaría en estrecha relación con el equipamiento tecnológico. Este equipamiento de espacios no sólo estaría en función del desarrollo de actividades relacionadas con el procesamiento de recursos en puntos específicos del paisaje, sino también con una planificación en la duración o recurrencia de tareas en los sectores de médanos. La misma planificación y recurrencia también se registra en los sectores elevados por sobre el fondo de cuenca (terrazas, cerros y bardas) que la circundan donde se encuentran las estructuras de piedra, en este caso vinculada con actividades de carácter funerario. Estas particularidades del registro arqueológico de la cuenca lacustre baja se destacan si se las enmarca en una escala espacial más amplia (Cassiodoro 2005).

La utilización de determinados puntos de la cuenca de los lagos Salitroso, Posadas y Pueyrredón y la reutilización de los mismos puede vincularse con un pautamiento en el uso del espacio. El conocimiento detallado del paisaje determina que el manejo de sus distintos elementos no sea azaroso, sino que esté en relación con la toma de decisiones respecto a la implementación de los asentamientos en zonas específicas (Schofield 1991). Así, por ejemplo, los médanos ofrecen muy buenas condiciones de reparo para el establecimiento de ocupaciones residenciales, mientras que las terrazas y lomadas son visibles y tienen disponibilidad de materias primas para la construcción de estructuras de entierro. El patrón recurrente de la ocupación de los mismos podría deberse a la existencia de una baja flexibilidad en la ubicación de espacios residenciales (Binford 1978), tanto en una escala espacial a nivel de cuenca como en una escala regional amplia. La recurrencia y el uso específico del espacio dan cuenta de la existencia de una circulación y movilidad pautada.

Una segunda expectativa arqueológica propuesta considera que el registro tecnológico del área deberá presentar una alta diversidad artefactual, en función del amplio espectro de actividades realizadas. 
En este caso, el patrón tecnológico de la cuenca apoya ampliamente la expectativa planteada. En las concentraciones de superficie se ha registrado una alta riqueza artefactual lítica. Un 79\% de las concentraciones tienen más de 10 categorías artefactuales representadas y los valores promedios del índice de riqueza son muy superiores a los registrados en otros contextos como los de la meseta alta de Pampa del Asador, donde los valores promedio de $\mathrm{H}$ son de 0.517 (Cassiodoro 2008). Esta alta riqueza artefactual sólo en parte es explicada por el tamaño de la muestra de los conjuntos y puede ser interpretada como el desarrollo de diferentes tipos de actividades vinculadas con la obtención y procesamiento de recursos y la producción de tecnofacturas. Cabe destacar que los valores promedio del índice de riqueza de la cuenca del lago Salitroso son muy similares a los registrados en Cerro de los Indios 1 (Guraieb 1999), sitio propuesto como de agregación de grupos (Aschero et al. 1999).

A esta diversidad de artefactos formatizados debe sumarse la existencia de distintos tipos de desechos de talla. Los mismos, principalmente, señalan tareas relacionadas con la extracción de formas base, actividades de descortezamiento inicial de guijarros, formatización final y mantenimiento de artefactos. La presencia de tiestos cerámicos en un $47 \%$ de las concentraciones artefactuales de superficie establece que la riqueza artefactual de las mismas sea aún mayor. Respecto a esta tecnología, se ha especificado que el proceso de manufactura se habría desarrollado en la cuenca (Cassiodoro y Tchilinguirian 2007; Cassiodoro 2008). Dado que el ciclo de producción de la cerámica puede llevar varios días, o por lo menos un mayor número que los que insume la tecnología lítica, su manufactura local podría ser un indicador de mayor permanencia de los grupos cazadores- recolectores o una porción del grupo en un lugar (Eerkens et al. 2002).

Por otro lado, no todas las concentraciones artefactuales de superficie son iguales. Algunas tienen tamaños menores, no presentan artefactos de molienda y cerámica, tienen una menor riqueza artefactual y clases artefactuales como núcleos y puntas de proyectil están más representadas. Esta variabilidad entre las concentraciones puede dar cuenta de la existencia, no sólo de espacios destinados a la ocupación residencial, sino también de sectores en los que se habrían desarrollado actividades más especificas, como por ejemplo la obtención de algún recurso en particular. Este tipo de variabilidad también se registra en relación con los espacios ubicados entre concentraciones que tienen una baja riqueza artefactual y una estructura artefactual diferente. Una utilización residencial y permanente de la cuenca no implicaría necesariamente una homogeneidad entre los conjuntos, por el contrario, no sólo es esperable una variabilidad en las tareas realizadas en cada una de las concentraciones, sino también entre ellas.

Se ha establecido la alta frecuencia de estructuras de piedra que básicamente constituyen entierros humanos en chenques. Los mismos se distribuyen en 17 puntos específicos que circundan la cuenca del lago Salitroso. Junto con las estructuras de entierro registradas en la costa norte de Santa Cruz (Castro y Moreno 2000) y en Puerto Ibáñez en Chile (Reyes 2001, Reyes 2002), la cuenca en estudio forma parte de una de las concentraciones de entierros humanos más relevantes de Patagonia. De esta manera, otra de las características del uso del espacio de la cuenca del lago Salitroso se vincula con la constitución de áreas formales de entierro, lo cual es coincidente con una ocupación residencial (Goñi et al. 2000-2002).

De la distribución y composición del registro arqueológico del lago Salitroso surge una clara segregación espacial entre las áreas de entierro y las destinadas a actividades domésticas. Como ha sido mencionado anteriormente, ambas actividades se localizan en distintos sectores de la cuenca y presentan estructuras artefactuales asociadas diferentes. Más allá de este claro patrón, también existe variabilidad al respecto. Por un lado, se encuentra el caso de SAC 3 que se caracteriza por ser una de las concentraciones artefactuales de superficie más extensa y con una alta riqueza artefactual, en cuyas inmediaciones se registra el único chenque en el fondo de cuenca (Goñi y Barrientos 2004). Por otro lado, en la superficie de algunos de los entierros que corresponden a SAC 2 se recuperó una alta cantidad y variedad de material lítico (Cassiodoro 2008).

En resumen, a partir de las características de la tecnología de la cuenca lacustre baja en estudio se puede establecer la existencia del desarrollo de variadas actividades en distintos sectores de la misma. Estas tareas no sólo se vinculan con la manufactura y mantenimiento de herramientas y tecnofacturas, la obtención y procesamiento de recursos y la 
preparación y almacenamiento de alimentos sino también con la realización de actividades de carácter funerario. Es de destacar que la mayor diversidad de los conjuntos artefactuales y la presencia de áreas con una alta frecuencia de entierros humanos han sido propuestas como indicadores de una menor movilidad residencial en contextos cazadores- recolectores (Shott 1986; Jones et al. 1999; Jones et al. 2003; Goñi et al. 2000- 2002; Veth 2005; entre otros). De esta forma, el carácter de las actividades desarrolladas explicaría parte de la variabilidad tecnológica de la cuenca en estudio. Dichas actividades son concordantes con el rol residencial propuesto para la misma y una ocupación más permanente.

Diferentes investigaciones tecnológicas centradas en los análisis microscópicos dan cuenta de las posibles limitaciones y dificultades que pueden surgir de establecer la funcionalidad de los conjuntos a partir de un análisis meramente tecno-morfológico (Álvarez 2004). Consideramos que parte de la diversidad de un conjunto está vinculada con las conductas que las produjeron (Shott 1989) y que una aproximación tecno-morfológica constituye un acercamiento a las mismas.

Cabe resaltar que otras líneas de evidencia como la información bioarqueológica y los análisis arqueofaunísticos también aportan información respecto el rol residencial de la cuenca (Aragone et al. 2004; Cassiodoro et al. 2004; Aragone 2007; García Guráieb et al. 2007a; Re y Aragone 2007).

Como ha sido señalado en otra oportunidad, dentro de la cuenca de los lagos Salitroso- PosadasPueyrredón existe variabilidad con respecto al rango de actividades llevadas a cabo (Cassiodoro et al. 2004; Re 2006; Re y Aragone 2007). En función de la frecuencia de chenques, el tamaño, densidad $y$ riqueza de las concentraciones artefactuales y las particularidades del registro arqueofaunístico, se ha propuesto que el sector oeste (lago Posadas) de la cuenca tendría un componente más logístico que el sector este (lago Salitroso). Esta variabilidad refuerza el rol marcadamente residencial del lago Salitroso.

Una última expectativa tecnológica vinculada con la reducción de la movilidad y el rol residencial y permanente de la cuenca se relaciona con las materias primas. De acuerdo con la disponibilidad de recursos minerales en el área, la mayor permanencia en la ocupación y la existencia de un uso complementario de otros ambientes a través de movimientos logísticos es esperable una alta diversidad de materias primas utilizadas. Se ha establecido que en el registro arqueológico de la cuenca del lago Salitroso, se encuentran representadas varias materias primas utilizadas en la tecnología tanto lítica como cerámica (Cassiodoro y Tchilinguirian 2007). Aunque en baja frecuencia, también existen tecnologías manufacturadas en metal, valvas y vidrio en las estructuras de entierros humanos, ampliando la variabilidad de materias primas utilizadas en el área.

Consideramos que esta variabilidad puede ser explicada en función de distintos factores. En primer lugar, parte de la diversidad está relacionada con las características de la base de recursos minerales de la cuenca en estudio. Como fuera mencionado, en la misma se encuentran disponibles diferentes materias primas líticas y cerámicas.

En segundo lugar, es esperable que cuanto más tiempo un grupo permanezca en determinado espacio, mayor uso realizará de las materias primas que se encuentran en el mismo (Roth 2000; Jones et al. 2003). De esta forma, parte de la variabilidad observada en el registro arqueológico puede relacionarse con la conjunción de dos factores: la disponibilidad de variadas materias primas y la movilidad residencial reducida propuesta para la cuenca.

A su vez, se ha planteado que un patrón de asentamiento relativamente estable implica la realización de movimientos de carácter logísticos y estacionales para el aprovisionamiento de recursos (Goñi 2000), lo cual permite el acceso a materias primas que no se encuentran disponibles en la cuenca. Éste es, fundamentalmente, el caso de la obsidiana que constituye una de las materias primas líticas más utilizadas en el lago Salitroso. La alta representación en los conjuntos de superficie de la cuenca lacustre de la materia prima cuya fuente se localiza en Pampa del Asador, establece la vinculación entre ambos espacios. En el mismo sentido también pueden considerarse algunas variedades de rocas silíceas.

De igual modo, la presencia de elementos manufacturados en valvas de origen marino, en metal y vidrio en los entierros humanos dan cuenta de las posibilidades de acceder (de manera directa o indirecta) a materias primas no disponibles en una escala regional. Al respecto, se ha planteado que una mayor frecuencia de materiales exóticos al área de estudio puede constituir la manifestación de la 
existencia de redes regionales más amplias y recurrentes (Veth 2005) y que la distancia desde donde se obtienen materiales puede incrementarse con un aumento en la duración y predictibilidad de las ocupaciones (Kuhn 1995). En síntesis, la realización de movimientos logísticos también contribuye a la variabilidad de materias primas utilizadas observada en el registro arqueológico.

Finalmente, se considera que las propiedades para la talla y los modos de acción específicos a los instrumentos confeccionados con diferentes materias primas líticas (Ratto 1991; Nami 1992) también pueden estar incidiendo en parte de la variabilidad. El desarrollo de una variada gama de actividades en la cuenca podría estar involucrando diferentes herramientas manufacturadas con materiales cuyas propiedades resulten acordes al modo de acción de las mismas, generando, en consecuencia, variabilidad en el registro arqueológico. De todas formas, esta selección es posible en función de una alta variedad de materias primas disponibles.

\section{CONSIDERACIONES FINALES}

En el transcurso de más de 10 años de investigación en la cuenca del lago Salitroso se ha aportado información desde distintas líneas de evidencia para la discusión del modelo de poblamiento tardío propuesto para el área (Goñi 2000; Goñi y Barrientos 2004). En este trabajo se buscó profundizar y ampliar la información tecnológica presentada en otras oportunidades (Goñi et al. 2000-2002; Cassiodoro et al. 2004). Consideramos que la caracterización general de la composición artefactual de la cuenca del lago Salitroso constituye un aporte más a dicha discusión. Las características de la distribución de la tecnología, la diversidad artefactual, los tipos de artefactos formatizados, los tipos de desechos de talla y las materias primas representadas en los diferentes conjuntos de superficie de la cuenca resultan de interés para la evaluación de la utilización de los distintos espacios por los grupos cazadores- recolectores durante el Holoceno tardío, momento que se caracteriza por una tendencia a la desecación ambiental progresiva, en una escala regional amplia. La profundización en el análisis de estas variables permite ampliar dicha discusión (Cassiodoro 2008).

La información tecnológica aquí presentada es una contribución a la evaluación de los modelos de poblamiento propuestos para Patagonia (Borrero 1989-1990; Goñi 2000). Por un lado, de manera particular, se otorgan argumentos a favor del modelo de poblamiento tardío planteado para el área de estudio, ya que distintos aspectos del registro tecnológico analizado pueden ser explicados en términos de una reducción de la movilidad residencial, un nucleamiento de los grupos y un uso diferencial del espacio ocurrido a partir de un descenso de la humedad regional. Por otro lado, al enmarcar las características del registro tecnológico estudiado dentro de un contexto regional mayor, se ofrece información útil a las diferentes etapas del proceso de poblamiento de Patagonia propuesto por Borrero (1989-1990), principalmente a la referida a la ocupación efectiva del espacio.

\section{AGRADECIMIENTOS}

A la Universidad de Buenos Aires que a través del otorgamiento de una beca Estímulo y su continuación en una beca Doctoral permitió que me dedicara de lleno a esta investigación. A su vez, mediante los proyectos UBACyT-FI 062, F072, F065 y F031 se pudieron efectuar las tareas de campo y los análisis de laboratorio. Agradezco a Rafael Goñi por sus comentarios y dirección y a todos los miembros del equipo que participaron en el relevamiento de campo. A los evaluadores de este trabajo y a Anahí Re. Muy especialmente a la hospitalidad y amistad brindada por la familia García de la Estancia Sierra Colorada.

\section{BIBLIOGRAFÍA}

ÁLVAREZ, M. 2004. ¿A qué responde la diversidad instrumental? Algunas reflexiones a partir del análisis funcional de materiales líticos de la costa Norte del Canal de Beagle. En: Contra Viento y Marea. Arqueología de Patagonia. Comp.: T. Civalero, P. Fernández y G. Guráieb. pp. 29-44. INAPL-SAA, Buenos Aires.

ARAGONE, A. 2007. Análisis faunístico comparativo entre los conjuntos óseos de médanos del lago Posadas y parapetos de Pampa del Asador. Tesis de licenciatura, FFyL UBA, Buenos Aires. MS

ARAGONE, A., T. BOURLOT, G. CASSIODORO y A. RE. 2004. Análisis comparativo del registro arqueológico en médanos del interior de Santa Cruz. Miradas. Trabajos de las V Jornadas de Jóvenes Investigadores en Ciencias 
Antropológicas. Coord.: M. Carballido, C. Pisarello y A. Re. pp. 327-345. INAPL, Buenos Aires.

ASCHERO, C. 1975 rev. 1983. Ensayo para una clasificación morfológica de los instrumentos líticos aplicada a estudios tipológico comparativos. Informe al CONICET, Buenos Aires. MS.

ASCHERO C., M. DE NIGRIS, M. J. FIGUERERO TORRES, G. GURÁIEB, G. MENGONI GOÑALONS y H. YACOBACCIO. 1999. Excavaciones recientes en Cerro de los Indios 1, Lago Posadas (Santa Cruz): nuevas perspectivas. En: Soplando en el Viento. Actas de las III Jornadas de Arqueología de la Patagonia. pp. 269-286. Edición INAPL- UN de Comahue.

BELLELLI C., G. GURÁIEB y J. GARCÍA. 1985-1987. Propuesta para el análisis y procesamiento por computadora de desechos de talla lítica (DELCO-desechos computarizados). Arqueología Contemporánea 2(1):36-53. Buenos Aires.

BERNAL, V., S. GARCÍA GURÁIEB y P. GONZÁLEZ. 2004.

Perfiles de mortalidad de las muestras de restos óseos humanos procedentes del área del lago Salitroso (provincia de Santa Cruz). En: Contra Viento y Marea. Arqueología de Patagonia. Comp.: T. Civalero, P. Fernández y G. Guráieb, pp. 361-373. INAPL-SAA, Buenos Aires.

BINFORD, L. 1978. Evidence for differences between residential and special purpose sites. En: Nunamuit Etnoarchaeology. pp. 488-497 Academic Press, New York

BINFORD, L. 1979. Organization and formation processes: looking at curated technology. Journal of Anthropological Research 35(3):255-273

BORRERO, L. 1989-1990. Evolución cultural divergente en la Patagonia austral. Anales del Instituto de la Patagonia 19:133-139. Punta Arenas, Chile.

CABRERA, A. y A. WILLINK 1980. Biogeografía de América Latina. Monografía $N^{\circ} 13$, OEA.

CARR, P. 1994. The organization of technology: impact and potential. En: The Organization of North American Prehistoric Chipped Stone Tool Technologies. Ed.: P. Carr. International Monographs in Prehistory Archaeological Series 7:1-8. Ann Arbor.

CASSIODORO, G. 2005. Equipamiento tecnológico del espacio en la cuenca del lago Salitroso (Santa Cruz). En: Entre Pasados y Presentes. Trabajos de las VI Jornadas de Jóvenes Investigadores en Ciencias Antropológicas. Coord.: A. Cetti, A. Re, D. Rindel y P. Valeri. pp. 210223. INAPL, Buenos Aires.

CASSIODORO, G. 2008. Movilidad y uso del espacio de cazadores-recolectores del Holoceno tardío: estudio de la variabilidad del registro tecnológico en distintos ambientes del noroeste de la provincia de Santa Cruz. Tesis de Doctorado, FFyL, UBA, Buenos Aires. MS

CASSIODORO, G., A. ARAGONE y A. RE. 2004. Más allá de los chenques...Registro arqueológico de sitios a cielo abierto en la cuenca de los lagos Salitroso-PosadasPueyrredón. En: Contra Viento y Marea. Arqueología de Patagonia. Comp.: T. Civalero, P. Fernández y G. Guráieb. pp. 325-338. INAPL-SAA, Buenos Aires.

CASSIODORO, G. y S. GARCÍA GURÁIEB. 2009. Análisis del registro tecnológico y osteológico de los entierros humanos del Holoceno tardío del lago Salitroso (Santa Cruz). En: Arqueología de Patagonia: una mirada desde el último confín. Ed: M. Salemme, F. Santiago, M. Alvarez, E. Piana, M. Vázquez y E. Mansur. pp. 613-628. Editorial Utopías, Ushuaia.

CASSIODORO, G y P. TCHILINGUIRIAN. 2007. Análisis petrográficos de cerámicas en el noroeste de la provincia de Santa Cruz. En: Arqueología de Fuego- Patagonia. Levantando piedras, desenterrando huesos...y develando arcanos. Ed.: F. Morello, M. Martinic, A. Prieto y G. Bahamonde. pp. 839-852. CEQUA, Punta Arenas.

CASTRO, A. y J. MORENO. 2000. Noticia sobre enterratorios humanos en la costa norte de Santa Cruz-PatagoniaArgentina. Anales del Instituto de la Patagonia: Serie Cs. Hum. 28: 225-231, Punta Arenas.

DE NIGRIS, M., M. J. FIGUERERO TORRES, A. G. GURÁIEB y G. MENGONI GOÑALONS. 2004. Nuevos fechados radiocarbónicos de la localidad de Cerro de los Indios 1 (Santa Cruz) y su proyección areal. En: Contra Viento y Marea. Arqueología de Patagonia. Comp.: T. Civalero, P. Fernández y G. Guráieb. pp. 537-544. INAPL-SAA, Buenos Aires.

EBERT, J. y T. KOHLER. 1988. The theoretical basis of archaeological predictive modeling and a consideration of appropriate data-collection methods. En: Quantifying the Present and Predicting the Past: Theory, Method and Application of Archaeological Predictive Modeling. Ed.: W. Judge y L. Sebastian, pp. 97-171 Bureau of Land Management, Colorado.

EERKENS, J., H. NEFF y M. GLASCOCK. 2002. Ceramic production among small-scale and mobile hunters and gatherers: a case study from the Southwestern Great Basin. Journal of Anthropological Archaeology 21: 200-229.

ESPINOSA, S. y R. GOÑI. 1999. Viven! Una fuente de obsidiana en la Pcia. de Santa Cruz. Soplando en el Viento. Actas de las III Jornadas de Arqueología de la Patagonia. pp. 177-188 Edición INAPL-UN de Comahue. 
GARCÍA Guráieb, S., P. GONZÁLEZ y V. BERNAL. 2007a. Estructura de sexo y edad de la muestra de restos humanos del Holoceno tardío del Lago Salitroso (Santa Cruz, Argentina). En: Arqueología de Fuego- Patagonia. Levantando piedras, desenterrando huesos...y develando arcanos. Ed.: F. Morello, M. Martinic, A. Prieto y G. Bahamonde. pp. 367-374. CEQUA, Punta Arenas.

GARCÍA GURÁIEB, S., R. GOÑI y L. BOSIO. 2007b. Lesiones traumáticas en un entierro del lago Salitroso (Santa Cruz, Argentina) En: Arqueología de FuegoPatagonia. Levantando piedras, desenterrando huesos...y develando arcanos. Ed.: F. Morello, M. Martinic, A. Prieto y G. Bahamonde pp. 375-380 CEQUA, Punta Arenas.

GONZÁLEZ, M. 1992. Paleoambientes del Pleistoceno Tardío/ Holoceno Temprano en la cuenca de los lagos Belgrano y Burmeister (47 $40^{\prime} / 48^{\circ}$ Sur, $72^{\circ} 30^{\prime}$ Oeste. Santa Cruz). Informe Técnico $\mathrm{N}^{\circ}$ 9:1-7. Fundación C. Caldenius.

GOÑI, R. 2000. Arqueología de momentos históricos fuera de los centros de conquista y colonización: un análisis de caso en el sur de la Patagonia. Desde el País de los Gigantes. Perspectivas Arqueológicas en Patagonia. pp. 283-296. UNPA, Río Gallegos.

GOÑI, R. 2000-2002. Fechados radiocarbónicos y registro arqueológico en la cuenca de los lagos Salitroso/Posadas (Santa Cruz). Cuadernos del Instituto Nacional de Antropología y Pensamiento Latinoamericano 19: 666-668.

GOÑI, R. y G. BARRIENTOS. 2000. Estudio de chenques en el lago Salitroso, pcia. de Santa Cruz. Desde el País de los Gigantes. Perspectivas Arqueológicas en Patagonia. pp. 161-175. UNPA, Río Gallegos.

GOÑI, R. y G. BARRIENTOS. 2004. Poblamiento tardío y movilidad en la cuenca del lago Salitroso. En: Contra Viento y Marea. Arqueología de Patagonia. Comp.: T. Civalero, P. Fernández y G. Guráieb. pp. 313-324. INAPL-SAA, Buenos Aires.

GOÑI, R., G. BARRIENTOS y G. CASSIODORO. 2000-2002. Condiciones previas a la extinción de las poblaciones humanas del sur de Patagonia: una discusión a partir del análisis del registro arqueológico de la cuenca del lago Salitroso. Cuadernos del Instituto Nacional de Antropología y Pensamiento Latinoamericano 19: 249-266

GURÁIEB, G. 1998. Cuáles, cuánto y de dónde: tendencias temporales de selección de recursos líticos en Cerro de los Indios I (Lago Posadas, Santa Cruz). Arqueología 8:77-99. FFyL, Buenos Aires
GURÁIEB, G. 1999. Análisis de la diversidad en los conjuntos instrumentales líticos de Cerro de los Indios 1 (lago Posadas, Santa Cruz). Relaciones de la Sociedad Argentina de Antropología XXIV: 293-306, Buenos Aires.

JONES, G., C. BECK, E. JONES y R. HUGHES. 2003. Lithic source use and paleoarchaic foraging territories in the Great Basin. American Antiquity 68(1): 5-38.

JONES, T., G. BROWN, M. RAAB, J. MCVICKAR, G. SPAULDING, D. KENNETT, A. YORK y P. WALKER. 1999. Environmental imperatives reconsidered. Demographic crises in Western North America during the Medieval Climatic Anomaly. Current Anthropology 40:137-170

JONES, G., D. GRAYSON y C. BECK. 1983. Artifact class richness and sample size in archaeological surface assemblages. En: Lulu Linear Punctated: Essays in Honor of George Irving Quimby, Ann Arbor: Anthropological Papers 72: 55-73, University of Michigan.

KUHN, S. 1995. Mousterian Lithic Technology. An Ecological Perspective. Princeton University Press. Princeton, New York.

LANATA, J. 1996. La diversidad instrumental en el norte de península Mitre, Tierra del Fuego. Arqueología 6: 159197. FFyL, Buenos Aires.

MELTZER, D., F. LEONARD y S. STRATTON. 1992. The relationship between sample size and diversity in archaeological assemblages. Journal of Archaeological Science 19:375-387.

NAMI, H. 1992. El subsistema tecnológico de la confección de instrumentos líticos y la explotación de los recursos del ambiente: una nueva vía de aproximación. Shincal 2:33-53.Universidad Nacional de Catamarca.

PIELOU, E. 1977. Mathematical Ecology. John Wiley and sons, Nueva York.

RATTO, N. 1991. Elección de rocas y diseño de artefactos: propiedades físico-químicas de las materias primas líticas de Inca Cueva 4 (Jujuy, Argentina). Actas XI Congreso Nacional de Arqueología Chilena. Vol. II: 121-137, Santiago.

RE, A. 2006. Uso del espacio en el oeste de la cuenca de los lagos Pueyrredón, Posadas y Salitroso (provincia de Santa Cruz): una aproximación tecnológica. Tesis de Licenciatura. FFyL UBA, Buenos Aires. MS

RE, A. y A. ARAGONE. 2007. El lejano oeste: uso del espacio en las costas del lago Posadas y en la margen sur del lago Pueyrredón (provincia de Santa Cruz). En: Arqueología de Fuego- Patagonia. Levantando piedras, desenterrando huesos...y develando arcanos. Ed.: F. Morello, M. Martinic, A. Prieto y G. Bahamonde. pp. 623-636. CEQUA, Punta Arenas. 
REYES, O. 2001. Enterratorios indígenas en el curso inferior del valle del Río Ibáñez, Región de Aisén. Boletín de la Sociedad Chilena de Arqueología 31:61-64

REYES, O. 2002. Funebria indígena en el curso inferior del valle del Río Ibáñez, margen occidental de la estepa centropatagónica (XI Región de Aisén). Anales del Instituto de la Patagonia: Serie Cs. Hum. 30:87-101, Punta Arenas.

ROTH, B. 2000. Obsidian source characterization and huntergatherer mobility: an example from the Tucson Basin. Journal of Archaeological Science 27: 305-314

SCHOFIELD, A. 1991. Artefact distributions as activity areas: examples from South-East Hampshire. En: Interpreting Artefact Scatters: contributions to ploughzone archaeology. Ed. A. J. Schofield, pp. 117-128. Oxbow Monograph 4.

SHANNON, C. y W. WEAVER. 1949. The Mathematical Theory of Comumunication. Urbana, The University of Illinois Press

SHOTT, M. 1986. Technological and settlement mobility: an ethnographic examination. Journal Anthropological Research 42:15-51
SHOTT, M. 1989. Diversity, organization and behavior in the material record. Ethnographic an archaeological examples. Current Anthropology 30(3):283-315.

STINE, S. 1994. Extreme and persistent drought in California and Patagonia during mediaeval time. Nature, vol. 369:546-549.

STINE, S. 2000. On the Medieval Climatic Anomaly. Current Anthropology 41(4):627-628

STINE, S. y M. STINE. 1990. A record from Lake Cardiel of Climate Change in Southern America. Nature 345(6277):705-708

VETH, P. 2005. Cycles of aridity and human mobility risk minimization among late Pleistocene foragers of the western desert, Australia. En: Desert Peoples. Archaeological Perspectives. Ed.: P. Veth, M. Smith and P Hiscock, pp. 100-115. Blakwell publishing

WINTERHALDER, B. y E. SMITH. 1992. Evolutionary ecology and the social sciences. En: Evolutionary Ecology and Human Behavior. Ed.: E. Smith y B. Winterhalder. pp. 3-23. Aldine de Gruyter, New York.

ZAR, J. 1974. Biostatistical Análisis. Nueva Jersey Englewood Cliffs Prentice Hall. 